\title{
Preclinical Imaging: an Essential Ally in Modern Biosciences
}

\author{
Lídia Cunha $\cdot$ Ildiko Horvath $\cdot$ Sara Ferreira $\cdot$ Joana Lemos • \\ Pedro Costa $\cdot$ Domingos Vieira $\cdot$ Dániel S. Veres $\cdot$ Krisztián Szigeti · \\ Teresa Summavielle $\cdot$ Domokos Máthé $\cdot$ Luís F. Metello
}

\begin{abstract}
Translational research is changing the practice of modern medicine and the way in which health problems are approached and solved. The use of small-animal models in basic and preclinical sciences is a major keystone for these kinds of research and development strategies, representing a bridge between discoveries at the molecular level and clinical implementation in diagnostics and/or therapeutics. The development of high-resolution in vivo imaging technologies provides a unique opportunity for studying disease in real time, in a quantitative way, at the molecular level, along with the ability to repeatedly and noninvasively monitor disease progression or response to treatment. The greatest advantages of preclinical imaging techniques include the reduction of biological variability and the opportunity to acquire, in

continuity, an impressive amount of unique information (without interfering with the biological process under study) in distinct forms, repeated or modulated as needed, along with the substantial reduction in the number of animals required for a particular study, fully complying with 3R (Replacement, Reduction and Refinement) policies. The most suitable modalities for small-animal in vivo imaging applications are based on nuclear medicine techniques (essentially, positron emission tomography [PET] and single photon emission computed tomography [SPECT]), optical imaging (OI), computed tomography (CT), magnetic reso-nance imaging (MRI), magnetic resonance spectroscopy imaging (MRSI), and ultrasound. Each modality has intrinsic advantages and limitations. More recently, aiming to over-come the inherent limitations of each imaging modality, multimodality devices designed to provide complementary information upon the pathophysiological process under study have gained popularity. The combination of high-resolution modalities, like micro-CT or micro-MRI, with highly sensi-tive techniques providing functional information, such as micro-PET or microSPECT, will continue to broaden the horizons of research in such key areas as infection, oncology, cardiology, and neurology, contributing not only to the understanding of the underlying mechanisms of disease, but also providing efficient and unique tools for evaluating new chemical entities and candidate drugs. The added value of small-animal imaging techniques has driven their increasing use by pharmaceutical companies, contract research organi-zations, and research institutions.
\end{abstract}




\section{Introduction}

Translational research is changing the practice of modern medicine and the way in which health problems are approached and solved. Its implementation is the basis for real science-based medicine ('from the researcher's bench to the patient's bedside'), being hailed as 'the' right direction for a more sustainable future, since it benefits from multidisciplinary, multiphase, and multisectorial projects to achieve solutions for health-related problems [1]. Nowadays, the use of small-animal models in basic and preclinical sciences is considered a major keystone for research and development strategies in biosciences [2]. It represents a bridge between discoveries at the molecular level and clinical implementation in diagnostics or therapeutics. This is of crucial importance for several reasons: on one hand, knowing the role of a molecule in a disease model in vitro may not mean that its role and molecular interactions in vivo are fully understood, while, on the other hand, human experimentation is not possible in most cases due to ethical and logistical issues [3]. Simultaneously, increasing efforts in the development of high-resolution in vivo imaging technologies are also providing unique opportunities to study disease noninvasively and, in many cases, quantitatively, at the molecular level, along with the ability to repeatedly and non-invasively monitor disease progression or response to treatment [4]. The combination of basic science knowledge and the new and more advanced animal models of disease with highly developed imaging tools enables the shortening of project length, improving the level of confidence in the obtained results and revenues and cost effectiveness. Moreover, the preclinical validation of drug targeting is driving the use of small-animal in vivo imaging technologies in drug development [3, 5]. Smallanimal imaging is being used in a wide variety of lines of research, especially in infection, inflammation, oncology, cardiology, and neurosciences.

In oncology, non-invasive in vivo imaging of tumors can be valuable in evaluating a number of parameters, including primary tumor mass/volume [6], number and location of metastases [4, 7, 8], glucose metabolism [9], tumor cell proliferation [10], gene expression [11-13], expression of membrane antigens [14], tumor angiogenesis [15-17], hypoxia [18], and apoptosis [19, 20].

Cardiovascular imaging is one of the most promising applications of molecular imaging, allowing the early detection of disease, risk stratification, monitoring the outcomes of innovative therapies [21, 22], and characterization of atherosclerotic plaques [23-26].

Imaging the brain in small animals remains one of the most challenging applications due to the small size of brain structures, the complex kinetics, and the relatively low tracer uptake [27]. However, cerebral blood flow, glucose uptake, density of neurotransmitter receptors and transporters, and drug occupancy studies have been successfully measured [28].
This paper undertakes a concise and critical review of the currently available modalities and emerging imaging technologies for in vivo small-animal imaging. Furthermore, the contribution and relative importance of each modality, including its advantages and limitations, is outlined. Finally, some considerations related to animal imaging facilities, including main concept design and workflow, is briefly covered. Examples of applications in the neuroscience field are mentioned, to better illustrate some of the ideas argued throughout the text.

\section{Small-Animal Imaging Modalities}

Zanzonico has provided a good definition of small-animal or preclinical imaging, stating that "it constitutes a way of assessing biological structures and function in vivo by noninvasive means, allowing the collection of quantitative information, both in health and disease states" [29]. That these techniques are non-invasive renders longitudinal studies possible, making it feasible to screen the entire spectrum of disease process, from disease onset to progression and therapy monitoring. The greatest advantages of imaging techniques are the reduction of biological variability (each animal works as its own control), paralleled by the substantial reduction in the number of animals required for a particular study [29] (Fig. 1). Moreover, the study of complex interactions between the physiological/ biochemical processes that occur in biological systems is only possible with intact animals in which variables related to immunological, nutritional, or hormonal aspects are present, as well as systemic responses, which it is not possible to fully evaluate with cell- or tissue culture-based methods or in ex vivo systems. In fact, other more complex, invasive, or time-consuming procedures, such as dissection, fixation, and sectioning, might be avoided [30]. As most small-animal imaging techniques are the same as those used in the clinical setting, results are easily translatable to humans [29, 31].

However, many challenges are still to be overcome when imaging a 20 - to 30 -g mouse as compared with a $70-\mathrm{kg}$ human, such as the size of the subject, the total volume to be imaged, the spatial resolution needed to obtain adequate anatomical and/or functional data as well as the total time spent acquiring a set of images [31]. The major challenge is to obtain a signal-to-noise ratio as high as possible and to localize the imaging probe as precisely as possible, while maintaining a good temporal resolution, using the minimum amount of molecular probe [32].

Among the different imaging modalities, nuclear medicine-based techniques (positron emission tomography [PET] and single photon emission computed tomography [SPECT]), optical imaging (OI), computed tomography 


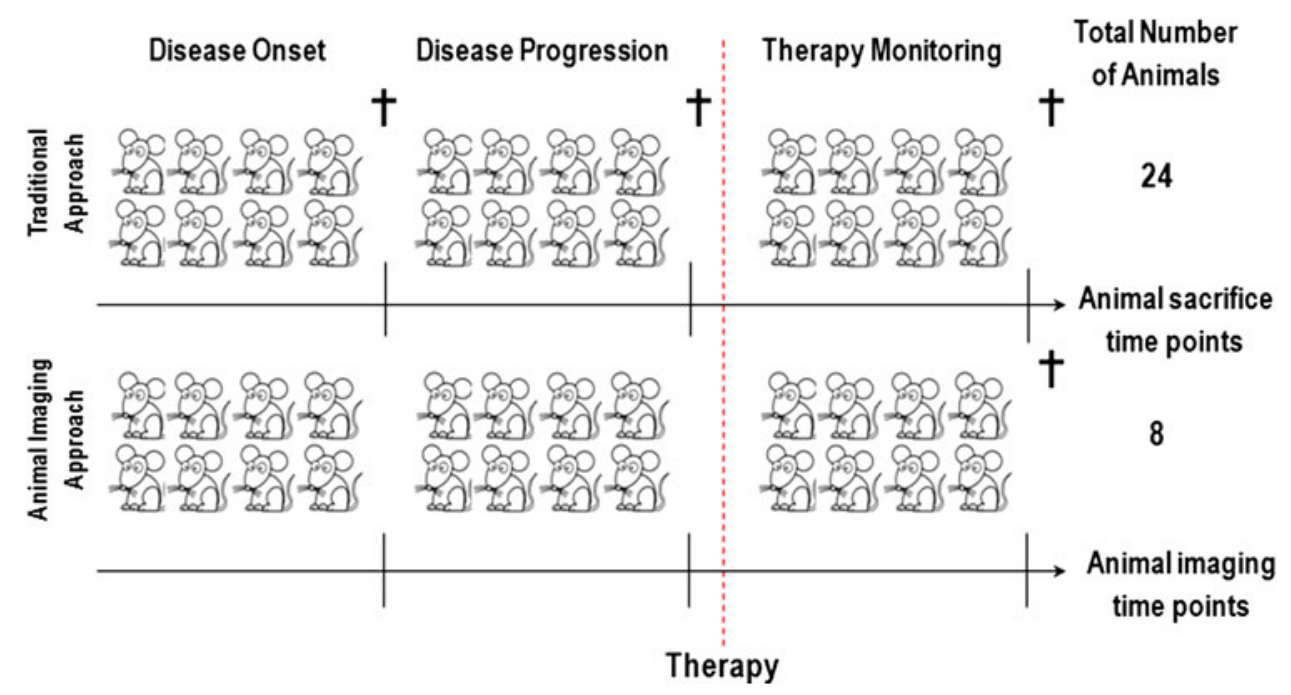

Fig. 1 Comparison between the number of animals used in traditional approaches and in small-animal imaging approaches. The traditional approach requires that a group of animals be sacrificed at each time point, making it impossible to study a single animal serially over time, and necessitating the use of a drastically greater number of animals to obtain statistical significance. The greater the number of time points required for a certain study, the greater the number of animals to be used, in practice limiting the number of time points to

(CT), magnetic resonance imaging (MRI), magnetic resonance spectroscopy imaging (MRSI), and ultrasound are the most suitable for non-invasive in vivo imaging, with the use of important pillars such as genomics, proteomics, and nanotechnologies [33].

Before dedicated devices were commercially available, small-animal imaging was performed in clinical instrumentation. Although the acquired images lacked proper resolution and sensitivity, it represented the first steps, which were not only an important contribution to demonstrating the valuable information that could be obtained from these techniques but was also the crucial impulse for the development of specific instrumentation that fulfill the need for improved equipment performance (namely, better spatial resolution, sensitivity, and tissue contrast). These improvements were related to imaging software and hardware (such as stronger magnetic fields and improved gradient fields and coils in MRI, reduction of ring diameter and detector-element size in PET, development of special pinhole collimators and multi-pinhole acquisition in SPECT, increase of X-ray flux and reduction of the focal spot in CT, and increase in ultrasound frequency) [29].

The existing imaging technologies differ essentially in their physical basis and in the type of information supplied (Table 1). Of critical importance are parameters such as spatial and temporal resolution, penetration depth, energy needed for image generation (ionizing or non-ionizing radiation), availability of molecular probes, and the respective detection threshold [32, 34]. be studied. Conversely, when using small-animal imaging techniques, the same group of animals is studied over the entire length of the study, significantly reducing the total number of animals to be used, without compromising the statistical significance and adding the possibility of having more time points, always without increasing the number of animals. Moreover, traditional techniques are low throughput and time consuming compared with small-animal imaging techniques

\subsection{Micro-SPECT}

SPECT and PET techniques are based on the tracer principle laid down by the Hungarian Nobel Laureate, George von Hevesy, which involves the injection of tiny amounts of radioactive tracers and the external assessment of their biodistribution by appropriate detectors. SPECT is able to detect $<10^{-10}$ molar of tracer molecules in vivo with submillimeter $(0.5-0.7 \mathrm{~mm})$ resolution, allowing the quantification of the molecular processes in which radioactive tracers are involved [35].

The use of multi-pinhole collimation has become the standard in most preclinical SPECT systems, resulting in great improvements, not only in spatial resolution but also in system sensitivity [36]. Sensitivity can be improved by increasing the number of pinholes and/or the angular sampling for a fixed field of view (FOV) [30, 37]. However, the overlapping projections from the distinct pinholes may constitute the main limitation in multi-pinhole collimator design, as the 'multi-plexing' phenomenon occurs, creating uncertainty about which pinhole a certain photon may have passed through. Therefore, the sensitivity advantage might be mitigated by the added pinholes and reconstruction artifacts may eventually arise [36]. Consequently, the use of a high-sensitivity, non-multiplexing, multi-pinhole approach might be considered a step forward.

Improvements in several system components have also been introduced, namely, new crystal materials in 
Table 1 Summary of general properties of diagnostic imaging modalities

\begin{tabular}{|c|c|c|c|}
\hline Imaging modality & Physical basis & Information supplied & Clinical use \\
\hline Positron emission tomography (PET) & $\begin{array}{l}\text { Gamma-radiation (derived from } \\
\text { positron emission) }\end{array}$ & Tracer uptake & Yes \\
\hline $\begin{array}{l}\text { Single photon emission computed } \\
\text { tomography (SPECT) }\end{array}$ & Gamma-radiation & Tracer uptake & Yes \\
\hline Optical imaging $(\mathrm{OI})$ & Light emissions (ex: fluorescence) & Probe uptake & $\begin{array}{l}\text { Yes (image-guided } \\
\text { surgery only) }\end{array}$ \\
\hline Computed tomography (CT) & $\mathrm{X}$-rays & Tissue density & Yes \\
\hline Magnetic resonance imaging (MRI) & Magnetic properties & Tissue composition & Yes \\
\hline Ultrasound (US) & $\begin{array}{l}\text { Sound reflection of high-frequency } \\
\text { sound waves }\end{array}$ & $\begin{array}{l}\text { Internal movements and flows, } \\
\text { differences of tissues }\end{array}$ & Yes \\
\hline
\end{tabular}

detectors, including thallium-doped cesium iodide $[\mathrm{CsI}(\mathrm{Tl})]$, sodium-doped cesium iodide $[\mathrm{CsI}(\mathrm{Na})]$, and cerium-doped lanthanum bromide $\left[\mathrm{LaBr}_{3}(\mathrm{Ce})\right]$, which offer improved light output and energy resolution and positionsensitive photomultiplier tubes (PMT). Finally, improvements in electronics have not only enhanced sensitivity, but also considerably reduced the overall equipment size and cost [30, 36, 37]. Some preclinical SPECT systems incorporate semi-conductor materials, such as cadmium zinc telluride (CZT) or silicon, as direct converters of gamma rays to electric signals [3, 38]. These detectors offer excellent spatial resolution, as low as $0.38 \mathrm{~mm}$ [39] and energy resolution, particularly important for low-energy radionuclides (as iodine-125) or dual isotope applications [3].

Although the use of scintillation crystals coupled to PMT is still very common, along with position estimation based on Anger logic principles, recent years have evidenced hardware progress that has been the basis of a drastic increase in available computational power. This has allowed the introduction of more sophisticated algorithms in data processing, essentially iterative algorithms, as the maximum-likelihood estimation and resolution recovery advanced solutions, thus improving global performance of systems [36]. Table 2 summarizes the main features of commercially available SPECT systems.

Single photon (SPECT) emitters are well suited for radiolabeling a variety of molecules, including endogenous biomolecules, such as peptides, antibodies, hormones, and selectins. These biomolecules are relatively large, making their diffusion into tissues, and blood clearance, low. This favors the use of radionuclides with longer half-lives (hours) in order to broaden the temporal window of observation, in contrast to positron emitters (with minutes of physical halflife) [35]. In neurosciences, drug-occupancy studies are often performed to evaluate the selectivity of a drug to specific brain regions. Our group used ${ }^{123} \mathrm{I}$-iodobenzamide $\left({ }^{123} \mathrm{I}-\mathrm{IBZM}\right)$ to evaluate dopamine receptor (D2R) occupancy in $\mathrm{C} 57 \mathrm{BL} / 6 \mathrm{~J}$ mice, before and after the administration of a dopamine agonist drug that induces the displacement of striatal ${ }^{123}$ I-IBZM binding, resulting in a decreased specific uptake ratio in the striatum (Fig. 2).

\subsection{Micro-PET}

PET is a well established clinical and preclinical imaging technology based on the application of compounds labeled with positron-emitting radioisotopes to image and measure several biochemical and physiological processes [40]. Due to its molecular, non-invasive, and non-destructive intrinsic nature, combined with the ability to study many biochemical/biological processes in vivo, it is universally recognized as a fundamental tool for biomedical research [41]. The possible fields of application for PET are quite broad, ranging from oncology to neurology and cardiology, among others, with less expression, but yet very relevant for infection and inflammation imaging.

The most commonly used positron emitters are ${ }^{11} \mathrm{C},{ }^{13} \mathrm{~N}$, ${ }^{15} \mathrm{O}$, and ${ }^{18} \mathrm{~F}$, but other isotopes such as ${ }^{60,61,64} \mathrm{Cu},{ }^{68} \mathrm{Ga}$, ${ }^{76} \mathrm{Br},{ }^{94 \mathrm{~m}} \mathrm{Tc},{ }^{89} \mathrm{Zr}$, or ${ }^{124} \mathrm{I}$ are gaining increasing attention and/or being tested for clinical applications. In fact, peptides labeled with ${ }^{68} \mathrm{Ga}$ have already demonstrated very promising results in the assessment of neuroendocrine tumors [42]. ${ }^{89} \mathrm{Zr}$ is well suited for the labeling of monoclonal antibodies, since more time for optimal biodistribution and tumor targeting is usually required [43]. Similarly, ${ }^{124}$ I has demonstrated higher efficacy in lesion detection and the ability to provide lesion-specific dosimetry in the context of differentiated thyroid cancer [44].

Although these radionuclides allow the study of many physiological and biochemical processes, they are difficult to obtain for many research groups, given their production method (mainly cyclotron-based) and the relative complexity and low yield quite often inherent to most of those 'non-traditional' (so 'non-optimized') synthesis processes. Additionally, their relatively short physical half-life makes distribution over long distances impractical or very expensive [45]. Even greater development in the 
Table 2 Features of some commercially available micro-SPECT scanners

\begin{tabular}{|c|c|c|c|c|c|c|c|}
\hline Manufacturer & Equipment & Detector & $\begin{array}{l}\text { Spatial } \\
\text { resolution }(\mathrm{mm})\end{array}$ & $\begin{array}{l}\text { Best sensitivity value } \\
(\mathrm{cpm} / \mathrm{MBq})\end{array}$ & $\begin{array}{l}\text { Collimation (no. of } \\
\text { pinholes) }\end{array}$ & $\begin{array}{l}\text { Multimodality } \\
\text { options }\end{array}$ & $\begin{array}{l}\text { Source of } \\
\text { information }\end{array}$ \\
\hline $\begin{array}{l}\text { Bruker }(\mathrm{ex}- \\
\text { carestream) }\end{array}$ & Albira & $\mathrm{CsI}(\mathrm{Na})$ & 0.6 & 1.000 & 9 & PET and CT & $\begin{array}{r}\text { Company } \\
\text { website }\end{array}$ \\
\hline Mediso & NanoSPECT & $\mathrm{NaI}(\mathrm{Tl})$ & 0.66 & $>4.000$ & 36 & CT & [113] \\
\hline Mediso & $\begin{array}{c}\text { nanoScan } \\
\text { SPECT }\end{array}$ & $\mathrm{NaI}(\mathrm{Tl})$ & 0.275 & $>10.000$ & 100 & CT or MRI & $\begin{array}{l}\text { Company } \\
\text { website }\end{array}$ \\
\hline MILabs & VECTor & $\mathrm{NaI}(\mathrm{Tl})$ & 0.38 & $>525$ & 75 & PET and/or CT & [114] \\
\hline Siemens & Inveon & $\mathrm{NaI}(\mathrm{Tl})$ & 0.5 & 2118 & 1 & PET and/or CT & [115] \\
\hline
\end{tabular}

CsI $(\mathrm{Na})$ sodium-doped cesium iodide crystal, $C T$ computed tomography, $C Z T$ cadmium-zinc telluride solid state detector, $M R I$ magnetic resonance imaging, $\mathrm{NaI}(\mathrm{Tl})$ thallium-doped sodium iodide crystal, PET positron emission tomography, SPECT single photon emission computed tomography

production and availability of positron emitters is expected in the near future. Not only due to the huge amount of work by a large number of researchers developing their activities in order to produce an increasing number of agents based on the interesting biochemical and physical properties of some of the above-mentioned radionuclides, but also to the increasing number of fully equipped dedicated facilities, radiopharmaceutical production and distribution centers, essentially based on low-energy (the so-called 'medical') cyclotrons and their specially dedicated laboratories. In fact, due to the short physical half-lives of most positron emitters (from seconds to a few hours), the use of this imaging modality may require in situ radioisotope production and compound labeling, and consequently, the existence at close distance of a multidisciplinary highly skilled and specialized team, with knowledge from distinct scientific areas.

Figure 3 summarizes the main steps of a PET study, where the agent has been produced either locally or externally and then delivered to its destination of final use. In fact, a huge number of radiotracers can only be used if locally produced, so it is now considered ideal for a preclinical facility (in order to adequately develop its role) to be near a radiopharmaceutical production facility. This is indeed a highly complex and costly condition; however, recent advances in cyclotron technologies, namely the anticipated introduction of the 'tabletop cyclotrons' and its 'all-integrated and automatic radiochemistry' approaches (directly for an interesting number of radioisotopes, such as ${ }^{11} \mathrm{C},{ }^{15} \mathrm{O},{ }^{13} \mathrm{~N}$, beyond the "classical ${ }^{18} \mathrm{~F}$ ) might overcome those aspects in the very near future.

An ideal PET scanner designed for small-animal imaging would have the following characteristics: high spatial resolution (sub-millimeter range), high sensitivity, detector ring with a FOV optimized to the specific targeted animal size range, good temporal resolution, and multimodality imaging capability. Many commercially available microPET scanners have resulted from previously developed prototypes in academia [46, 47]. Nowadays, the main players are either companies specializing in the preclinical field or big companies known in the clinical imaging arena that also present preclinical imaging devices in their portfolio. Table 3 presents some examples of the devices available in the market and their respective features.

Micro-PET detector blocks are made of inorganic scintillators, such as lutetium oxyorthosilicate (LSO), lutetium-yttrium oxyorthosilicate (LYSO), or gadolinium orthosilicate (GSO), which have replaced the old bismuth
Fig. $2{ }^{123}$ I-I-iodobenzamide uptake in mouse striatum measured in single photon emission computed tomography - magnetic resonance imaging (SPECT-MRI) co-registration images. Transaxial section planes of the mouse brain, striatal uptake is high in the left panel, while displacement is imaged in the right panel with low striatal uptake of the dopamine transporter ligand
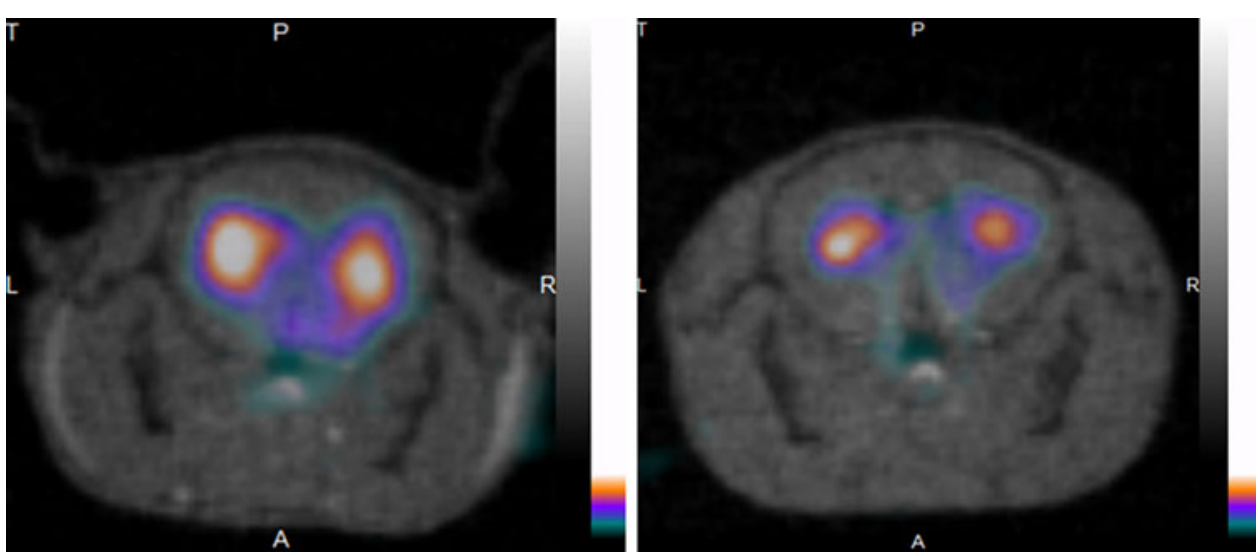
Fig. 3 Main steps of a positron emission tomography (PET) study using a locally produced agent (upper row) compared with an externally produced and locally distributed agent (bottom row)

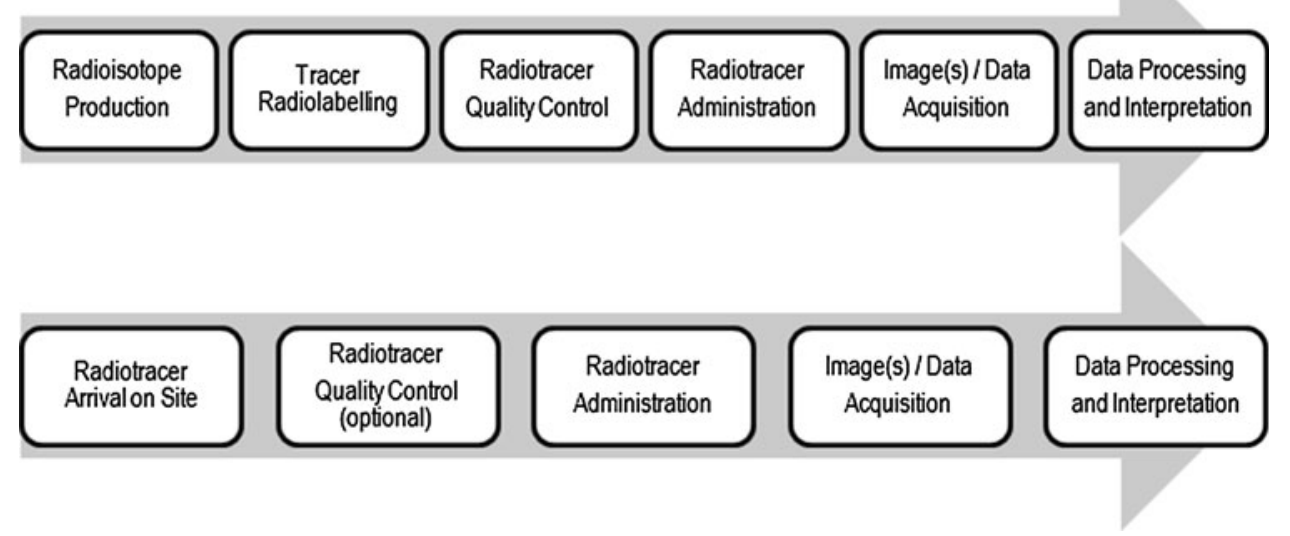

germanate (BGO) scintillators with their more favorable speed, light output, and detection efficiency [46, 48]. In comparison with clinical scanners, micro-PET devices provide much better sensitivity and spatial resolution. These scanners can achieve spatial resolution values of around $1.0 \mathrm{~mm}$ in reconstructed images, but only conjugating the application of radioisotopes with low energy positrons (from ${ }^{18} \mathrm{~F}$, for instance) with a state of the art equipment that could reach pixel size less than $1.2 \mathrm{~mm}$ and an intrinsic resolution of $0.7 \mathrm{~mm}$ [49]. In fact, the positron range, depending on positron energy, is a hard physical intrinsic barrier to PET resolution (thereby SPECT will always have higher resolution, due to the lack of anything similar to the 'positron range' in gamma-emitting isotopes).

Over the years, PET has been established as a powerful and reliable tool in the biomedical research arena. Yet, its utilization (concerning both the quantity and the diversity of available procedures) is highly dependent on the presence or not of an 'on site solution' for radiopharmaceutical production, since without it, PET imaging will be entirely dependent on the existence of a reliable radiotracer distribution network of fluorinated compounds, those which are the most easily and currently distributed. This is why one of the most useful and widely used parameters is glucose consumption in a variety of situations, for example, by a tumor or by brain tissue. Figure 4 exemplifies the use of ${ }^{18}$ F-FDG, a known glucose analog, for the assessment of mouse brain glucose uptake under control conditions and after drug preconditioning that induces a massive increase in glucose uptake by all regions.

Even considering these relevant potential limitations, the application of this imaging modality has been increasing considerably in industrialized countries, and is

Table 3 Features of some commercially available micro-PET scanners

\begin{tabular}{|c|c|c|c|c|c|c|c|}
\hline Manufacturer & Equipment & Crystal & $\begin{array}{l}\text { Transaxial } \\
\text { FOV }(\mathrm{cm})\end{array}$ & $\begin{array}{l}\text { Spatial resolution } \\
(\mathrm{mm})\end{array}$ & $\begin{array}{l}\text { Sensitivity } \\
(\%)\end{array}$ & $\begin{array}{l}\text { Multimodality } \\
\text { options }\end{array}$ & $\begin{array}{l}\text { Source of } \\
\text { information }\end{array}$ \\
\hline Bruker & Albira & LYSO & 8 & 1.3 & $3-9$ & $\begin{array}{l}\text { SPECT and/or } \\
\text { CT }\end{array}$ & [133] \\
\hline Mediso & $\begin{array}{l}\text { nanoScan } \\
\text { PM PET }\end{array}$ & LYSO & 12.4 & 0.7 & 9 & CT or MRI & [134] \\
\hline MILabs & VECTor $^{\mathrm{a}}$ & $\mathrm{NaI}(\mathrm{Tl})$ crystals & 8.2 & 0.75 & (Variable) & $\begin{array}{l}\text { SPECT and/or } \\
\text { CT }\end{array}$ & [135] \\
\hline Raytest & ClearPET & $\begin{array}{l}\text { LYSO and LuYAP in } \\
\text { double layer }\end{array}$ & 11 & 1.5 & 5 & CT or MRI & [136] \\
\hline Siemens & Inveon & LSO & 12.7 & 1.4 & 10 & $\begin{array}{l}\text { CT and/or } \\
\text { SPECT }\end{array}$ & [137] \\
\hline $\begin{array}{l}\text { Sofie } \\
\text { biosciences }\end{array}$ & Genisys4 & BGO & 4.5 & 1.4 & 14 & X-Ray & [138] \\
\hline
\end{tabular}

$B G O$ bismuth germanate, $C T$ computed tomography, FOV field of view, LGSO lutetium-gadolinium oxyorthosilicate, $L S O$ lutetium oxyorthosilicate, LUYAP cesium doped lutetium-yttrium orthoaluminate, LYSO lutetium-yttrium oxyorthosilicate, MRI magnetic resonance imaging, SPECT single photon emission computed tomography

${ }^{a}$ Due to special collimator design, VECTor might be seen as a high-energy collimated SPECT 
Fig. 4 Increased ${ }^{18}$ F-FluoroDeoxy-D-Glucose uptake in the mouse brain after drug treatment (bottom row) compared with normal glucose uptake in a non-treated mouse (upper row). PET/MRI (nanoScan ${ }^{\circledR} \mathrm{PM}$, Mediso, Hungary) images acquired under light isoflurane anesthesia. Coronal (left) and transverse slices (right) are shown. A anterior, $\mathrm{Cer}$ cerebellum, $C t$ brain cortex, $F$ feet direction, $H$ harderian glands, $L$ left, $M R I$ magnetic resonance imaging, $P$ posterior; PET positron emission tomography, $R$ right, $T h$ thalamus
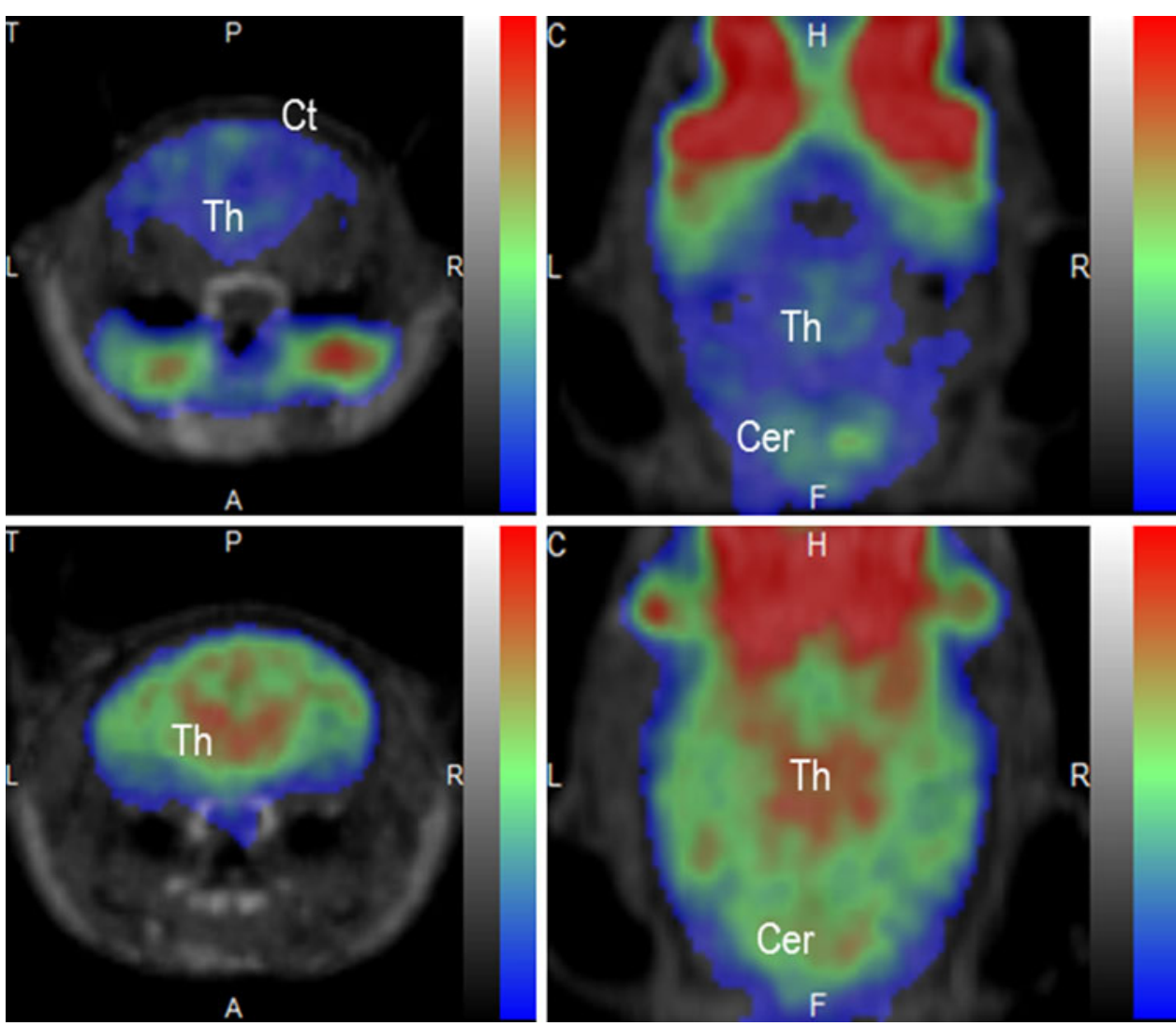

now ranked as the third most used single preclinical imaging technique, representing $20 \%$ of the preclinical imaging applications, just behind OI (with $28 \%$ only for the bioluminescence techniques) and MRI, with $23 \%$ [50].

\subsection{Micro-SPECT versus Micro-PET}

One of the major strengths of nuclear imaging techniques is the ability to image the same animal using a variety of tracers with different biological characteristics, providing information about the molecular affinity of the tracer for the distinct biological processes being studied.

Traditionally, PET imaging has been seen as the more accurate for quantification purposes due to its higher sensitivity. However, in recent years, development of SPECT systems has led to higher resolution and sensitivity capabilities, making it a very attractive option for quantitative in vivo imaging [51]. The leading advantages of PET include its higher detection efficiency and, when it is the case, the availability of such positron-emitting radionuclides as ${ }^{11} \mathrm{C},{ }^{13} \mathrm{~N},{ }^{15} \mathrm{O}$, and ${ }^{18} \mathrm{~F}$, which allows the labeling of many physiologically and biochemically interesting biomarkers that are involved in health and disease processes [52-54].

The most important hardware difference between PET and SPECT cameras is normally the absence of a collimator in PET, making it about tenfold more sensitive, as collimators reject many of the counts arising from the source [55]. However, PET has intrinsic limitations associated with positron range $(0.6-3 \mathrm{~mm})$, non-collinearity of photons, and random events that limit the spatial resolution of the system [27, 30, 56, 57]. This is why current smallanimal PET systems have a standardized spatial resolution of $1.55 \mathrm{~mm}$ (range of 1-2.2 mm) [4, 58], while typical SPECT systems offer improved resolution in the order of 0.35-0.7 mm [35, 59, 60].

The major advantages of SPECT are the ability to use a variety of radioactive agents based on radioisotopes with different energies, a relatively simple and stable chemistry allowing the synthesis of ligands on site, and their relatively long physical half-life, making them easily accessible for many research groups $[35,45]$ and suitable for use in investigational contexts where there is a need for longer periods for data acquisition (see Tables 4 and 5 to compare the most widely used PET and SPECT agents). Many of the SPECT agents can be obtained from central radiopharmacies, as they are widely used in clinical nuclear medicine. In many cases, if a desired radiopharmaceutical is not commercially available, a relatively simple laboratory setup is enough to produce it. Another advantage of smallanimal SPECT is its ability to image multiple radiotracers that emit different energy photons simultaneously, 
Table 4 Main characteristics of the most used SPECT radionuclides

\begin{tabular}{lllll}
\hline Radionuclide & Decay mode & Physical half-life & Photon energies- $\mathrm{keV}$ and abundances $(\%)$ & Specific activity $(\mathrm{GBq} / \mathrm{mol})$ \\
\hline${ }^{99 \mathrm{~m}} \mathrm{Tc}$ & Isomeric transition & 6.02 hours & $140(89)$ & $2.0-5.0 \times 10^{9}$ \\
${ }^{67} \mathrm{Ga}$ & Electron capture & 3.26 days & $93(36) ; 184(20) ; 296(16)$ & $1.3-2.7 \times 10^{6}$ \\
${ }^{123} \mathrm{I}$ & Electron capture & 13.2 hours & $159(83)$ & $12.3 \times 10^{7}-43 \times 10^{8}$ \\
${ }^{131} \mathrm{I}$ & Beta minus & 8.02 days & $284(6) ; 364(81) ; 637(7)$ & $222-327 \times 10^{6}$ \\
${ }^{111} \mathrm{In}$ & Electron capture & 2.80 days & $173(90) ; 245(94)$ & $60 \times 10^{6}$ \\
${ }^{201} \mathrm{Tl}$ & Electron capture & 3.04 days & $69(27) ; 71(46) ; 80(20)$ & $8.0 \times 10^{5}-8.0 \times 10^{6}$ \\
\hline
\end{tabular}

providing information from distinct biological processes at the same time, from the same model [53]. Finally, smallanimal SPECT studies generally cost less than other imaging methods, such as small-animal PET [3].

The mass effect of the injected dose is a very important, although often neglected, aspect of imaging small animals with radionuclides. In general, the amount of injected radioactivity does not scale with the animal size. When going from human to mouse, the volume resolution element is reduced from about $1 \mathrm{~cm}$ to $1 \mathrm{~mm}$. However, compared with clinical applications, spatial resolution requirements in small-animal imaging are much higher; the concentration of the injected tracer per gram of tissue is usually much higher in small animals, with a ratio of 20 times that in a human $[47,61]$. Moreover, in order to obtain a comparable number of counts per pixel, and thus a global spatial resolution between clinical and preclinical scanners, significantly higher doses of radioactivity per unit of body mass should be administered for imaging rodents in preclinical scanners. Accordingly, this leads to the administration of a higher amount of chemical compound per body mass, which may threaten the validity of the tracer principle (so implicating the occupancy of $<5 \%$ of the total available receptor sites) [62]. Another important constraint in small-animal imaging is the maximum injected volume, which should not exceed $10 \%$ of the total blood volume. Therefore, when considering a $20-\mathrm{g}$ mouse with a blood volume of $2 \mathrm{~mL}$, intravenous injections should ideally not exceed $200 \mu \mathrm{L}$ [63]. Considering all this, in order to obtain accurate and reproducible results, it is crucial that radioactive tracers have high specific activities, which is considerably more achievable with SPECT than with PET tracers [63] (Tables 4, 5).

Acquiring high-quality images of the mouse brain can be very challenging due to the low radiotracer uptake (typically below $1 \%$ of the administered activity) and to the small sizes of the structures to be imaged. Despite the high sensitivity of PET, SPECT has a clear added value in brain applications due to the higher spatial resolution (below $1.0 \mathrm{~mm}$ ) and the afore-mentioned ability to use longer-lasting radionuclides (Tables 2, 3, 4, and 5). Moreover, PET tracers usually have much lower specific activities than SPECT-labeled compounds, which may lead to significant receptor occupancy or eventually to pharmacological effects [3].

Although small-animal SPECT and PET images offer unique functional information at the molecular level, they are often difficult to interpret because of the lack of

Table 5 Main characteristics of the most used PET radionuclides

\begin{tabular}{lllllll}
\hline Radionuclide & $\begin{array}{l}\beta^{+} \text {Decay } \\
(\%)\end{array}$ & $\begin{array}{l}\text { Physical half-life } \\
\text { (minutes) }\end{array}$ & $\begin{array}{l}\text { Max positron energy } \\
(\mathrm{MeV})\end{array}$ & $\begin{array}{l}\text { Mean positron range in } \\
\text { water }(\mathrm{mm})\end{array}$ & $\begin{array}{l}\text { Production } \\
\begin{array}{l}\text { Specific activity } \\
(\mathrm{GBq} / \mathrm{mol})\end{array}\end{array}$ \\
\hline${ }^{11} \mathrm{C}$ & 99 & 20.4 & 0.98 & 1.12 & Cyclotron & $3.4 \times 10^{5}$ \\
${ }^{13} \mathrm{~N}$ & 99 & 10.0 & 1.19 & 1.44 & Cyclotron & $6.8 \times 10^{5}$ \\
${ }^{15} \mathrm{O}$ & 100 & 2.07 & 1.72 & 2.22 & $\mathrm{Cyclotron}$ & $3.4 \times 10^{6}$ \\
${ }^{18} \mathrm{~F}$ & 97 & 109.8 & 0.63 & 0.64 & Cyclotron $6.3 \times 10^{4}$ \\
${ }^{68} \mathrm{Ga}$ & 90 & 68.3 & 1.90 & 2.24 & Generator & n.a. \\
${ }^{82} \mathrm{Rb}$ & 96 & 1.3 & 3.15 & 2.80 & Generator & n.a. \\
${ }^{89} \mathrm{Zr}$ & 22,3 & 4708.8 & 8.97 & 1.18 & Cyclotron & $1.8-44.2 \times 10^{6}$ \\
${ }^{124} \mathrm{I}$ & 25 & 6048 & 3.16 & 2.80 & Cyclotron & $1.11 \times 10^{9 *}$ \\
\hline
\end{tabular}

* Data from Veit-Haibach et al. [121]

n.a. information not available, $P E T$ positron emission tomography 
correlation with anatomic structures or biologic landmarks. This lack of detailed anatomical information may be considered the major weakness of nuclear techniques, sometimes making it difficult to clarify the precise localization of tracer uptake and the delineation of regions of interest for quantitative analysis. This is an important issue, since the accuracy of quantitative analysis is dependent on a variety of factors, namely, the real size of the lesion/region, the intensity of tracer uptake, the image contrast, the attenuation of the gamma rays by the surrounding tissues or the scanner materials, the color scale, and the image intensity threshold (upper and lower limits) [64].

\subsection{Micro-CT}

Micro-CT is a morphological imaging technique that measures and compares differences in tissue densities. It is conducted at an almost microscopic level compared with a clinical CT system used for human imaging [65]. A typical micro-CT system consists of an X-ray tube and an X-ray detector assembled in opposing positions in a rotating gantry. Although the underlying physical principles of both preclinical and clinical scanners are the same, the former requires images with higher resolution. In this sense, special components had to be introduced, such as X-ray tubes with smaller focal spots and detectors with small elements, to produce sharp images. Micro-CT provides 3-dimensional (3D) tomographic data at microscopic spatial resolution $(50 \mu \mathrm{m})$ and sub-second temporal resolution $(50 \mathrm{~ms})$ [66] of soft tissues and bone structures by capturing hundreds of 2-dimensional (2D) projections from multiple angles around the animal $[30,54,65,67]$. This imaging modality has been useful in a number of applications, namely the assessment of skeletal and lung abnormalities, heart function, and tumor growth. Micro-CT is a robust technique for the quantitative evaluation of angiogenesis associated with solid tumors or ischemia [30,67]. Although $\mathrm{CT}$ is the preferred technique for lung imaging, due to the high contrast provided by air/tissue differences, in the preclinical context, imaging of such small and moving structures has been challenging [67]. To overcome this problem, respiratory gating devices have become commercially available over recent years, thus reducing breathing motion-related artifacts [54]. This has resulted in increased lung tissue image contrast, thus significantly improving image quality [65].

CT is entirely based on the quantification of X-ray attenuation by the tissues, meaning that subtle differences are very difficult to distinguish due to almost identical attenuation characteristics. In an attempt to overcome this limitation, contrast agents might be administered, enhancing CT sensitivity by highlighting either the vascular tree or specific areas of organs or tissues. The most commonly used CT contrast agents are iodinated water-soluble compounds, usually administered by intravenous injection [68]. These agents distribute exclusively in the extracellular fluid space and present very short biological half-lives due to fast clearance, which shortens the temporal window of observation and consequently, prevents their wider utilization [69]. The main advantages of micro-CT over other small-animal imaging modalities are the high spatial resolution images, with detailed morphological information and the time required for scanning (typically $10-15 \mathrm{~min}$ ). Nevertheless, the radiation burden associated with CT imaging, and the volume of contrast agents (used to enhance the contrast resolution) that it is possible to inject into a single animal (particularly mice), are potential limitations of which researchers should be aware. Generally, depending on the desired image contrast and resolution, modern scanners provide short acquisition times with resolution levels below $100 \mu \mathrm{m}$ and radiation doses in the range of $10-50 \mathrm{mGy}$ [70]. Researchers should always be aware that, as in the clinical context, a balance between image quality and animal irradiation must be found in order to meet As Low As Reasonably Achievable (ALARA) principles, thus avoiding unnecessary radiation exposure, which is particularly important when imaging series are required.

\subsection{Micro-MRI}

MRI is a non-ionizing 3D imaging technique that uses magnetic properties of tissues and their interactions with strong external magnetic fields. Due to its paramagnetic properties as well as its ubiquitous body distribution, hydrogen nucleus $\left({ }^{1} \mathrm{H}\right)$ from water molecules is most used in MRI imaging. Briefly, the underlying principle is that, when a sample within a magnetic field is subjected to a radio-frequency pulse, its protons absorb energy and generate a detectable signal during the relaxation phase that can be digitally encoded through magnetic field gradients to generate digital images. The strength of the signal is a function of the number of protons, and the differences in the microenvironment of those protons between tissues determine the appearance of the image [2, 5, 71, 72]. Water has a wide variety of biophysical magnetic signatures in tissues and organs, and a key to success in many experiments is optimizing experimental methods and parameters in order to enhance contrast between healthy and pathological tissue [2].

Micro-MRI provides morphological images with excellent contrast and spatial resolution $(100 \mu \mathrm{m})$, as well as information regarding tissue composition, perfusion, oxygenation, tissue elasticity, metabolism, and detection of molecular probes, within a single acquisition session without radiation exposure [73]. These attributes have 
contributed to the increasing popularity of MRI among scientists and hence the widespread utilization. Nowadays, this imaging modality represents about $23 \%$ of all smallanimal imaging procedures [50]. Compared with clinical scanners, preclinical MRI scanners require a tenfold increase in spatial resolution in each dimension $(\mathrm{X}, \mathrm{Y}, \mathrm{Z})$, resulting in signal reductions of at least 1,000 . To overcome this challenge, stronger magnets, specific receiver coils and radiofrequency receiver chains, and stronger gradient sets were introduced [74]. As the signal-to-noise ratio increases with the magnetic field, small-animal MRI scanners should be at least $4.7 \mathrm{~T}$ or, ideally, higher than 7.0 T, according some authors. In fact, dedicated smallbore MR devices can operate between 4.7 and $21 \mathrm{~T}$. However, they are very expensive and not widely available. Moreover, one should be aware that $1.0 \mathrm{~T}$ represents 20,000 times the magnetic field of the planet (so, 140,000 times for the above-mentioned 7.0 $\mathrm{T}$ devices) and even though, to our knowledge, there is no consistent evidence of physiological alterations induced by (extremely) high magnetic fields, such hypotheses should never be discarded, in our opinion.

If requirements concerning spatial and temporal resolution are not particularly strict, clinical scanners (operating typically between 1.5 and $3.0 \mathrm{~T}$ ) may be a suitable option as long as special radiofrequency coils and optimized pulse sequences are used [74, 75].

Currently, a variety of MRI techniques are available for the study of many biological processes. One example is diffusion-weighted imaging (DWI), based on Brownian motion, allowing the characterization of the movement of protons in tissues, mainly from the water molecule during an interval of time. Molecular diffusion in tissues is the result of the interaction of molecules with many 'barriers' such as other molecules, cellular organelles, fibers, and membranes. The molecular diffusion patterns provide information about tissue architecture and cellularity, since the higher the cell density (more barriers), the lower the diffusion will be, leading to high signal intensities in diffusion-weighted images and to low 'apparent diffusion coefficient' (ADC) values [76]. DWI has been used for tumor characterization in non-moving structures such as bones and the brain [77], being particularly sensitive for the early detection of brain ischemia [78].

Another MRI technique is the so-called 'functional MRI' (fMRI; also designated by blood oxygen level dependent [BOLD]), based on the conjunction of both neuronal activity and brain hemodynamics. fMRI essentially measures changes in blood oxygenation, which translate into changes in the magnetic field. This is possible because oxyhemoglobin and deoxyhemoglobin have distinct magnetic properties (the former is weakly diamagnetic, while the latter is paramagnetic), inducing inhomogeneities into the surrounding magnetic field. Thus, a decrease in the concentration of deoxyhemoglobin will increase image intensity. This technique is commonly used for brain activation studies [79].

Perfusion measurements are possible using two distinct MRI techniques: (i) the dynamic contrast-enhanced imaging (DCE-MRI), in which an intravenous bolus injection of a contrast agent is detected during its first passage through the organs and (ii) arterial spin labeling (ASL), in which the arterial blood water magnetization itself functions as an endogenous contrast agent [76]. The size of the contrast agent in DCE-MRI will determine its washout from the vascular space, making it possible to measure vascular permeability [76]. The ASL technique works better at high magnetic fields (3.0 T and above) and highly irrigated organs, such as the brain, the heart, muscles, and kidneys, with the advantage of not requiring contrast injection, allowing repeated measurements [76, 80-83].

MRI is now a well established modality for imaging the cardiovascular system, providing valuable information concerning structure, function, and perfusion, both at cellular and at molecular levels in mouse models of cardiovascular disease. It is also a powerful modality for the determination of the cardiovascular phenotypes of genetically engineered mice and the evaluation of novel targeted contrast agents (e.g. gadolinium, manganese, and super paramagnetic iron oxide nanoparticles [SPION]) $[53,54]$.

In oncology, MRI has been used extensively in the characterization of tumors in a wide variety of animal models, namely tumor growth and development. Due to its sensitivity to dynamic processes, it is an excellent tool to image tumor perfusion/angiogenesis and oxygenation. Moreover, the non-invasive and non-destructive nature of MRI is particularly advantageous in monitoring in vivo tumor growth and ablation in response to therapy, as it is possible to serially monitor individual subjects of a given cohort over an extended period of time [2, 71]. Micro-MRI has also been successfully applied in the assessment of musculoskeletal tissue structures such as tendons, cartilage, menisci, and ligaments (although the assessment of bone structure remains a challenge because only $15 \%$ of the bone volume is composed of water) [72].

\subsection{MRSI}

MRSI is a combination of magnetic resonance spectroscopy (MRS) and MRI, wherein the first modality provides physiological/biochemical information and the latter anatomical information. In reality, MRSI imaging consists of a hybrid system that provides and relates images in a given tissue or organ with the concentration of certain chemicals found in the same tissue or organ, providing information about tissue biochemistry. These chemicals are detectable 
by the MR process because they are composed of nuclei that have a magnetic moment (such as hydrogen $\left[{ }^{1} \mathrm{H}\right]$, carbon $\left[{ }^{13} \mathrm{C}\right]$, phosphorus $\left[{ }^{31} \mathrm{P}\right]$, and fluorine $\left[{ }^{19} \mathrm{~F}\right]$ ) [84]. Magnetic nuclei in a molecule are surrounded by other magnetic or charged particles with which they interact, causing a chemical shift, providing unique resonance frequencies for nuclei of different molecular groups. In fact, resonance frequencies of nuclear spins depend on their chemical environment, providing a resonance signature of the chemical structure, allowing the identification of the compounds present in the organ being studied [85]. The intensity of the spectral signals is related to the concentration of the compound in tissues, thus allowing quantitative approaches. In order to achieve a high signal-to-noise ratio, high magnetic fields are required (3.0 $\mathrm{T}$ and above) [86].

MRSI has had an established role as a clinical tool since it was approved by the US FDA in 1995 [87], allowing the detection of relatively small-sized molecules, usually in concentrations of $0.5-10 \mathrm{mM}$ [88]. Similarly to MRI, hydrogen-based MRS, which is also called 'proton MRS', has become increasingly popular due to the high natural abundance of protons, their high absolute sensitivity to magnetic manipulation, better spatial resolution [87] (compared with MRS based on other, less abundant, nuclei such as ${ }^{13} \mathrm{C}$ or ${ }^{19} \mathrm{~F}$ ), and relatively simple technique (that can be performed using clinical MRI systems with standard radiofrequency coils), as well as the abundant presence of these nuclei in most metabolites [87, 88]. The results of MRS are displayed as a spectrum, where the concentration of a metabolite is linearly proportional to its spectral peak area [89]. Although MRS can theoretically be performed in almost any tissue of the human/animal body, the brain has been the main target of MRS studies [87, 90], with $\mathrm{N}$-acetyl aspartate, choline, creatine, myo-inositol, lactate, lipids, glutamine, glutamate, and amino acids being the major compounds of interest [87, 89]. It has also been shown to be useful in the assessment of tumor metabolism in breast, brain, and prostate cancers. This modality can detect specific genetic and metabolic changes that occur in malignant tumors, being able to define different metabolic tumor phenotypes [91].

\subsection{Micro-CT versus Micro-MRI}

To conduct a legitimate comparison between preclinical CT and MRI, it is essential to understand that, despite both of these techniques providing mainly anatomical information, they have entirely distinctive applications and underlying principles. In fact, both of the techniques go far beyond the anatomical information they initially provided, autonomously evolving at not only an individual level, but also as a component of hybrid imaging techniques such as
PET-CT and PET-MRI. Generally, X-ray-based methods are less expensive and time consuming than MRI. MicroCT provides excellent contrast and spatial resolution of bone structures and MRI is more suitable for soft-tissue assessment due to its higher sensitivity and contrast, providing important information regarding the biological properties of tissues. The spatial resolution of micro-CT is better $(50 \mu \mathrm{m})$ than micro-MRI $(100 \mu \mathrm{m})$. Moreover, MRI does not involve ionizing radiation, which is often considered advantageous for longitudinal studies. MRI is also a very effective imaging method when it comes to readouts from a single scanning session, as many different facets of MRI-acquired data analysis and reconstruction exist. In fact, in its multiple facets, MRI is able to provide not only detailed anatomical information but also unique physiological data such as organ perfusion, blood velocity, blood volume, vessel permeability, molecule diffusion, oxygen consumption, and tissue chemical composition [76].

\subsection{Optical Imaging}

OI includes a variety of techniques that have in common the use of a set of light sources and respective sensing devices to capture the resulting photon distribution. These techniques can be classified according to the type of source-detector setting and the contrast mechanism applied. To improve image contrast, targeted fluorescent or activatable probes were developed, making it possible to measure the activity of the chosen molecular targets. Imaging of such probes involves the excitation of the probe at a certain wavelength and the detection of the specific signal emission at a significantly different wavelength. The most relevant OI techniques are bioluminescence, fluorescence, and near-infrared (NIR) fluorescence imaging [92]. Recent reports have also described OI techniques being used to image ultraviolet and visible light produced by radioactive materials-Cerenkov imaging [93].

For image acquisition, the animal must be placed in a light-tight imaging enclosure and the emitted light is then captured by a charged-coupled detector (CCD). In order to detect visible to near infra-red light emitted from the body, highly sensitive detectors have been produced; CCD detectors that are made of silicon crystals sliced into thin portions for assembly into integrated circuits [32]. CCD cameras operate by converting light photons at wavelengths between 400 and $1,000 \mathrm{~nm}$, that strike a CCD pixel with an energy of only $2-3 \mathrm{eV}$ [32].

The principle of bioluminescence imaging consists in the emission of visible photons at specific wavelengths based on reactions catalyzed by enzymes (luciferases) present in many organisms as protists, fungi, insects, 
bacteria, among other species [94]. Luciferases catalyze the oxidation of luciferins (substrate), creating non-reactive oxyluciferins and releasing photons of light [95]. Over the last decade, advances in molecular techniques have led to the isolation of many luciferase genes that have been used to build DNA vectors [94]. Currently, the most commonly used varieties of luciferase-luciferin are the firefly (Fluc) [95].

Highly sensitive imaging systems allow the quantitative detection of small numbers of cells or organisms that express luciferase as a transgene [31]. This technique requires the target cells to have been previously genetically transduced ex vivo in order to express the 'reporter' or luciferase gene. The luciferase substrate (luciferin) is then systemically administered and when it reaches the target cells, is oxidized by luciferase, emitting photons that are captured by CCD cameras [92].

A bioluminescence image is often shown as a color image that is superimposed on a gray-scale photographic image of the small animal using overlay and image analysis software. The main advantage of optical bioluminescence imaging is that it can be used to detect very low levels of signal (as there is almost no background light).

The principle of fluorescence imaging is different from that of bioluminescence. Here, an excitation light with a wavelength in the visible range of 395-600 $\mathrm{nm}$ illuminates the animal and a CCD camera captures an emission light of shifted wavelength. Cells can be either genetically transduced in order to express a fluorescence molecule as the green fluorescent protein (GFP) or a fluorescent probe like a fluorescence-labeled antibody may be systemically administered [32]. In NIR fluorescence imaging, light in the 700- to $900-\mathrm{nm}$ range is used to maximize tissue penetration and minimize autofluorescence from non-target tissues [96].

Diffuse optical tomography (DOT), a computational approach to OI, presents improved imaging performance as multiple source-detector pairs are used to produce 3D images with good sensitivity and spatial resolution, and accurate quantification and volumetric localization [97].

Nevertheless, effective resolution of bioluminescence, fluorescence, or NIR imaging is still suboptimal and the resulting images are not quantitative, because factors such as the scattering, attenuation, and dispersion of the emitted light as it penetrates tissue layers mean the captured signal is highly dependent on the depth of the tissue of origin (structures closer to the surface will appear brighter than deeper structures) [31]. A more recent approach to fluorescence imaging of deeper structures uses fluorescencemediated tomography [98], which uses multiple projections and measures light around the boundaries of the object to be imaged. After mathematical processing, reconstructed tomographic images can be obtained with a resolution of
1-2 $\mathrm{mm}$ and a fluorochrome detection threshold in the nanomolar range [32]. In contrast to planar images, these $3 \mathrm{D}$ images are quantitative, as the signal intensity is directly related to the local concentration of fluorochrome [98].

The photo-acoustic effect was first described by Alexander Bell in 1880, and photo-acoustic tomography (PAT) was more recently reported by Kruger [99]. It is an imaging modality that benefits from the advantages of pure OI or ultrasound imaging, without the major disadvantages of each technique. This is done by illuminating the sample with short pulses of laser beams and collecting the ultrasound waves generated by the photo-acoustic effect, which can be described briefly as follows. A fraction of incident light pulse energy is absorbed by the target tissue and converted into heat, causing a rise in the temperature and thus a thermal expansion of the object. This increase in pressure propagates as a sound wave, which can be detected externally as an acoustic signal [100]. PAT has undergone tremendous development in the past decade; in the near future, its main preclinical applications might include imaging of angiogenesis, tumor microenvironments, drug response, biomarkers, and gene activity [100].

OI based on high-energy beta particle-emitting radionuclides is the more recently discovered variant of this imaging modality, described for the first time by Robertson et al. [101], who showed that positron-emitting $\left(\beta^{+}\right)$radionuclides are able to produce visible light, consistent with Cerenkov radiation, with a continuous spectrum that is weighted towards the ultraviolet and blue bands of the electromagnetic spectrum. Cerenkov radiation is produced when charged particles move at very high speeds, in certain mediums, allowing the emission of optical photons, which can be captured by commercially available CCD cameras. Cerenkov radiation is produced in a continuous spectrum from the near ultraviolet through the visible spectrum [101]. Recent works published by Liu et al. [102] and Robertson et al. [101] demonstrated that, using the lowenergy window of light $(1.2-3.1 \mathrm{eV}, 400-1,000 \mathrm{~nm})$ resulting from beta particle-emitting $\left(\beta^{+}\right.$and $\left.\beta-\right)$ radionuclides, it is possible to produce images with ordinary OI equipment. The radionuclide agents that have already been tested include ${ }^{18} \mathrm{~F}$-FDG, $\mathrm{Na}^{18} \mathrm{~F}, \mathrm{Na}^{131} \mathrm{I},{ }^{90} \mathrm{YCl}_{3}$, and several ${ }^{90} \mathrm{Y}$-labeled peptides. The advantages of radionuclide OI/ Cerenkov imaging over the conventional OI techniques essentially relate to the fact that imaging probes are the same as those used in the clinical context, presenting a wide emission spectrum, making it possible to monitor the radioactive probe at different wavelengths with no need for excitation light. However, some limitations of this technique, such as limited tissue penetration and relatively poor quantification ability compared with PET and SPECT are common to other OI techniques [102]. The first human 
'Cerenkography' of a patient treated for hyperthyroidism with ${ }^{131}$ I was recently (2013) described by Spinelli et al. [103].

Overall, the most important advantages of OI are the high sensitivity, low cost, ease of handling, relatively high throughput, short acquisition time (typically 10-60 seconds), visualization of physiological and pathophysiological processes at the cellular and molecular level in vivo with high specificity, and the possibility of simultaneous imaging of at least six anesthetized mice [32, 104]. An additional advantage of $\mathrm{OI}$ is the fact that several probes with different spectral characteristics can be used for multichannel imaging.

\subsection{Ultrasounds}

Ultrasounds refer to sound waves with frequencies higher than 20,000 cycles/s $(\mathrm{Hz})$ that are not detectable by the human ear $[105,106]$. The underlying principle of ultrasound imaging resides in the use of the interaction of sound waves with living tissues/organs to produce images [106]. Diagnostic ultrasound commonly uses frequencies between 2 and $15 \mathrm{MHz}$ [106], while preclinical systems use higher frequencies $(20-50 \mathrm{MHz})$ to provide images with higher spatial resolution and an adequate penetration for anatomical and functional real-time information of the animal models [107].

Ultrasounds offer numerous specialized imaging formats and techniques that are used routinely in clinical and preclinical practices. Brightness mode (B-mode), motion mode (M-mode), spectral Doppler and color Doppler are the most common imaging formats. Ultrasound biomicroscopy (UBM), contrast-enhanced imaging, and transesophageal echocardiography (TEE) are three specialized ultrasound imaging techniques that have been used in rodents [106]. Doppler-based models are dynamic, realtime images used to determine the velocity of a moving tissue and to obtain quantitative structural and functional information from the target organ [106]. The Doppler effect is the apparent shift in sound-wave frequency that occurs when a sound wave is reflected by a moving target, mainly blood cells. The greater the Doppler shift, the greater the velocity of the blood flow [106]. Ultrasound Doppler is widely used in clinical practice to study the vascular system, namely to determine blood flow velocity and direction [108] and to image soft tissues.

Contrast agents (material with acoustic properties that differ from the tissues to be imaged) may be used to enhance image quality [105]. Molecular-targeted contrast agents may be used to evaluate biological processes at the molecular level [109]. The most common ultrasound contrast agents are microbubbles, which are small, stabilized gas-encapsulated microparticles $(<10 \mu \mathrm{m})$. After intravenous injection, they behave similarly to the red blood cells in the circulation, providing a strong reflective blood/gas interface [110].

Ultrasound biomicroscopy operates at higher frequencies (typically $40-200 \mathrm{MHz}$ ) $[105,110]$ and is able to acquire images using 2D B-mode, pulsed and continuous wave Doppler and color flow Doppler [50, 110]. This imaging modality has been applied in different contexts, namely the assessment of embryonic and eye development [111, 112], cardiology [113], and oncology [114].

Ultrasound imaging has several advantages over other imaging techniques: high spatial and temporal resolution and a rapid frame rate, allowing real-time and quantitative imaging; absence of ionizing radiation; low cost; broad availability; and minimal or no sedation is required [105, 109]. However, ultrasounds can cause bioeffects, including tissue heating and shock waves [105]. As reproducibility of the results is highly dependent on the operator, a well trained and skilled sonographer is required in order to obtain accurate, repeatable, and high-quality images [106]. Due to limitations in imaging bone and gas-filled structures, ultrasounds are not commonly used in the brain, spinal cord, and lung [110].

\subsection{Hybrid and Multimodality Imaging}

The limitations inherent to each imaging modality, be it from the technical point of view (low resolution or sensitivity) or applicability (availability of imaging probes), has driven the widespread combination of two or more modalities to image the same tissue or organ in the same animal [30]. Multimodality devices are designed to provide complementary information upon the subject/pathophysiological process being studied. The most common are the combinations between high sensitivity and functional modalities, such as PET and SPECT, with high spatial resolution and morphological techniques, such as CT and MRI. Multimodal imaging can be achieved either by acquiring the images in separate devices and performing image fusion later, using robust software, or acquiring images almost simultaneously in the same imaging device with dual- or trimodality. However, software approaches did not really meet expectations in terms of accuracy of image registration and fusion or ease of use (since it usually involves many steps and relies on the operator's visual accuracy), making image fusion a difficult task due to variations in animal positioning. Concerning the second approach, commercially available systems include the combination of PET-CT, PET-MRI, SPECT-CT, and, more recently, SPECT-MRI. Other possible combinations, such as PET-OI [115], SPECT-OI [116], CT-OI [117], CT-MRI [118], OI-MRI [119], are also under development. It is also possible to find in the market trimodality 
systems combining PET-SPECT-CT. Some groups are working on the development of such additional solutions as SPECT-CT-OI [120]. Multimodal combination has enabled some of the most important limitations of each imaging modality to be overcome when used alone. For instance, the combination of CT or MRI with PET or SPECT has allowed more precise localization of focal points of abnormal tracer uptake, which has contributed to more accurate diagnosis, in both the clinical and the preclinical setting [121, 122]. In addition, more recently, the introduction of new technologies, such as avalanche photodiodes (APD) coupled with scintillating crystals (PET or SPECT), lowered the limits of spatial resolution, improving the overall image quality while enhancing the overall sensitivity [123].

CT data can be used to derive a transmission map for object-specific attenuation correction. Precise attenuation correction has proven particularly beneficial in the clinical context $[122,124]$ as there is an improvement of image quality paralleled with more accurate quantification. Attenuation correction is more pertinent in SPECT applications than in PET, because of its intrinsic dependence on the unknown depth of the detected photons emanating from tissues [125]. Synchronous PET-MRI, the only physically available modality where image acquisition is truly simultaneous, was initially developed to improve spatial resolution of PET $[126,127]$. In fact, the positron range, well known to be an intrinsic limiting factor of PET resolution, decreases in the presence of strong magnetic fields. This aspect may be potentially advantageous in preclinical imaging, particularly when high-energy positron emitters are used in combination with strong magnetic fields ( $>4.5$ T) [128]. PET-CT and SPECT-CT became commercially available earlier than PET-MRI and have served as proof of concept that the combination of functional and morphological imaging techniques could represent more than the sum of the parts, revealing that PET-MRI and SPECT-MRI might be equally or even more advantageous, particularly for brain imaging (Fig. 5). In fact, MRI might be preferred over CT for a number of reasons: improved soft tissue contrast (particularly important in neuroimaging and abdominal and pelvic regions), no additional radiation exposure (important for longitudinal studies), and the possibility of obtaining supplementary functional information (perfusion, tissue oxygenation, etc.) [129].

In the past, a significant drawback of MRI was the inability to provide information for attenuation correction. Nevertheless, due to intense research in the field, several methods have been presented, while others still under development are showing very promising results (for a review, please see Greco et al. [110]). The clinical robustness of these methods is being tested in order to implement the best of them in the clinical routine in a near future [130].
The combination of PET or SPECT modalities with MRI has followed a more daring and completely different approach from that used for CT. The existing systems are based on the incorporation of PET or SPECT detectors inside MR magnets, aiming to acquire data at exactly the same time. Evidently, this has increased the complexity and presented significant technical challenges (essentially the electromagnetic interference between the two systems). A much simpler approach, the combination of nuclear techniques with $\mathrm{CT}$, is based on a tandem configuration in which images are acquired sequentially rather than simultaneously [129].

Multimodality imaging started with the development of software tools for image co-registration, followed by dedicated instrumentation development. Research has now been focused on the development of multimodality imaging probes, which are compounds that can provide contrast for more than one imaging modality (proteins, peptides, microbubbles, nanoparticles) [129]. Some exciting results are already being observed in this area, with microbubbles, traditionally used in ultrasound imaging, the lipid coat of which might be labeled with fluorescence and/or radionuclide agents and thus, used for multimodality imaging ultrasound-OI-PET/SPECT [131]. Multimodality imaging of small animals has considerably expanded the type of information available from a single in vivo experiment, allowing the visualization of in vivo cellular and metabolic processes without requiring animal sacrifice.

\section{Additional Considerations}

The use of animals for research purposes, under strict legal regulation, requires pre-approval of the physical infrastructures, the experimental protocols, the imaging devices, and biosafety and radiation protection procedures. Moreover, specialized and specific staff education and veterinary support must be assured. The creation of an animal imaging center must be carefully planned, primarily by previously determining the kind of research that will be held and which imaging modalities will be needed. The commercially available imaging devices are generally very expensive, ranging from $\$$ US100,000 (for OI systems) to more than \$US2,400,000 (for more sophisticated MRI systems). Prices will be even higher for multimodality or hybrid systems. To prevent inefficiencies and unnecessary increases in overall costs when initiating an imaging facility, workflow should be optimized. This includes the determination of which imaging devices to buy, how many, and their physical localization; which spaces need humidity, temperature, or pressure control (essentially negative or positive pressurized rooms); what are the special requirements concerning the power supply, radiation, and/or 

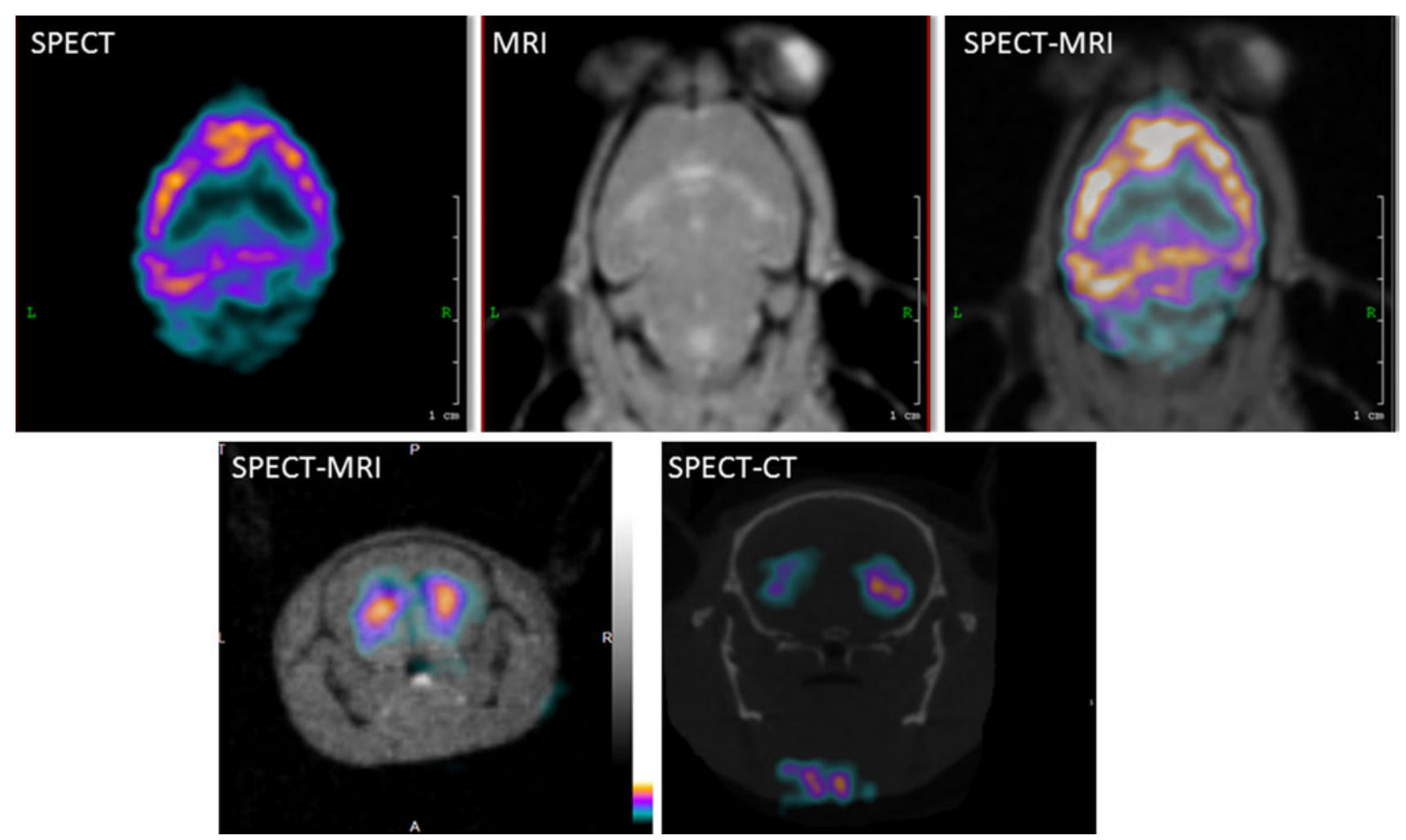

Fig. 5 Transverse slices of SPECT and MRI images of a mouse brain. SPECT was acquired using a specific agent for cortical benzodiazepine receptors $\left({ }^{23} \mathrm{I}-\mathrm{NNC13}-82431\right)$. The lack of anatomical information of SPECT acquisition is complemented with the information provided by MRI, in which the eyes, the olfactory bulbs and the first and second ventricles are shown. The multimodality SPECTMRI image provides information about functional benzodiazepine receptors from SPECT allied to good soft tissue contrast from the

magnetic shielding; biosafety conditions for immunocompromised animals; which areas will have restricted or controlled access; and which spaces need to be supplied with medical gases. The plan should also include space for animal husbandry, surgical, and quarantine areas; radiochemistry-specific spaces, including a dedicated decay room; an autoradiography and sectioning room; and a storage room. It is very important to define the different flows that may occur in the department (staff, animals, materials, and radiation sources) in order to avoid crosscontamination and unnecessary radiation exposure. The planning and design of an animal imaging facility is a complex task requiring the expertise of a multi-skilled team that may include architects, veterinarians, staff from the provider company (or companies), experienced imaging technologists, radiopharmacists, physicists, and engineers.

A practical requirement in preclinical imaging is that the animal remains still during the imaging procedure, which may often involve the administration of anesthetizing gas (the most widely used and consensual approach) or injectable drug cocktails, as well as the use of life- and
MRI (upper row). Multimodality SPECT-MRI and SPECT-CT images illustrating the increased soft tissue contrast of MRI modality compared with CT, emphasizing the added value of adding anatomical information from MRI to function information from SPECT in the context of brain studies. SPECT images were acquired with ${ }^{123} \mathrm{I}$ Iodobenzamide (bottom row). MRI magnetic resonance imaging, SPECT single photon emission computed tomography

comfort-support devices (electrodes and thermometer for heart rate and temperature monitoring, artificial ventilation, warming pads, etc.). Ideally, all animal-imaging devices should be equipped with appropriate immobilization apparatus, gaseous anesthesia system, barriers for immunocompromised animals, and temperature control. In the case of rotating gantries, care should be taken in order to allow the free rotation of the gantry while the animal remains still in the imaging bed [70]. Moreover, in the case of longitudinal studies where multiple doses of anesthesia may be required, the effect of dose and time-interval between doses should be considered in order to avoid misleading results.

When choosing the most appropriate imaging modality (or modalities) for a certain study, one must consider not only the scientific question to be addressed, but also the physical conditions and the possible logistic limitations for the implementation and execution of the experimental procedures. Data may be more easily transposed to humans if the chosen modalities for small-animal imaging have clinical homologs, thus reducing the gap between 


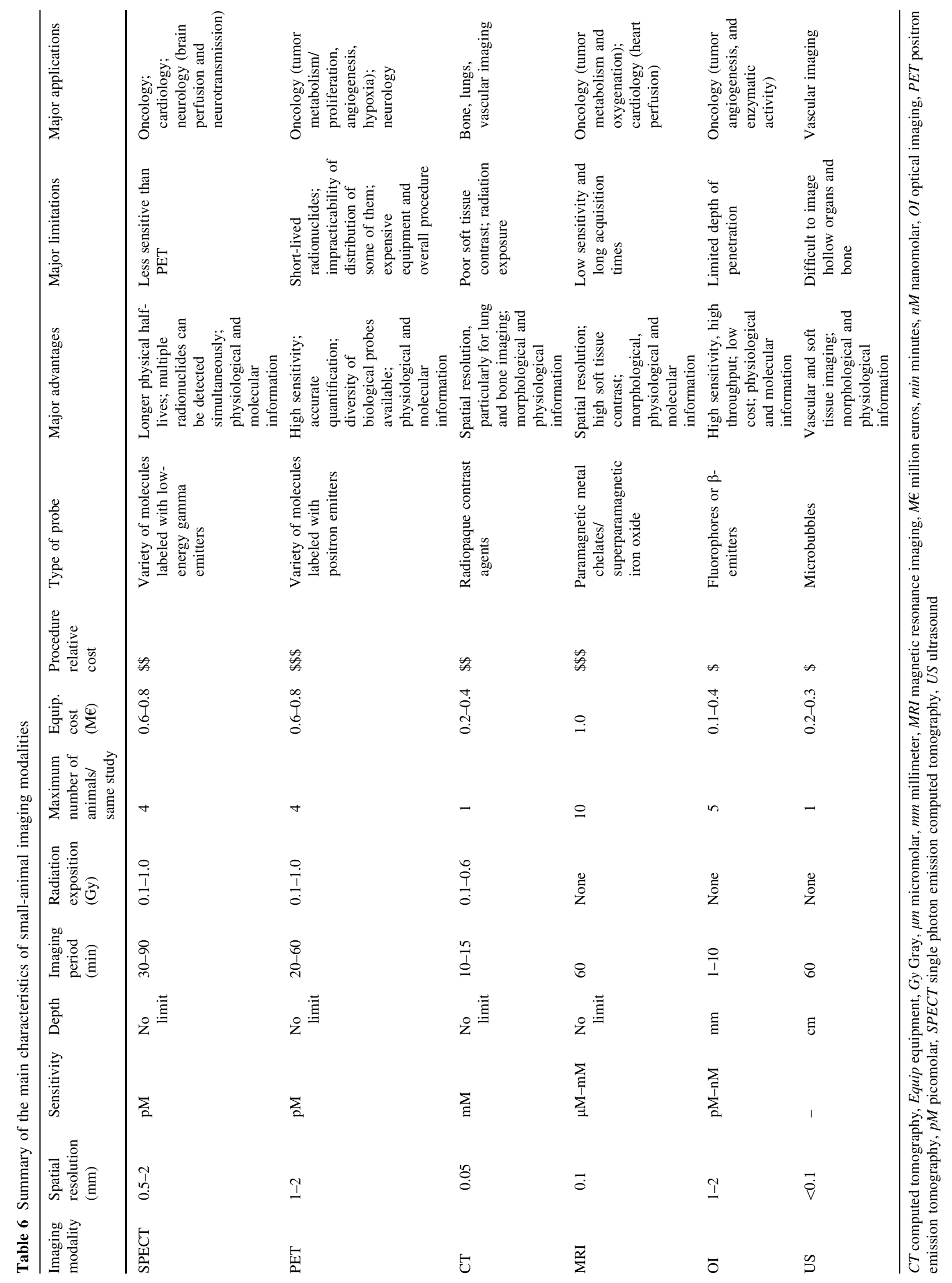


preclinical and clinical studies. In this sense, OI, despite its low cost and target-specific pharmacodynamics, may not be a suitable option if direct translation into clinical use is desired (with the exception of image-guided surgery so far). Nuclear techniques such as PET and SPECT provide valuable information at the molecular level about tracer pharmacokinetics and cellular metabolism. The wide variety of available molecular probes for nuclear imaging allows the study of an almost endless number of biological processes. When choosing PET or SPECT modalities, one has to consider several aspects such as tracer availability and related logistics, radionuclide physical half-life, specific activity of the tracer and the required temporal window for image acquisition. PET has the highest sensitivity, allowing more accurate quantification, but SPECT provides images with higher spatial resolution-it is adequate for longer studies and is usually less expensive than PET. Although CT and MRI both provide morphological information, each modality may be applied with different purposes: while $\mathrm{CT}$ provides maps for attenuation correction and increased contrast in bone structures, MRI provides excellent soft tissue contrast and additional functional information without involving the use of ionizing radiation. Often, the use of more than one imaging modality may be required. Although it will necessarily increase the overall costs, it might provide information that it would otherwise not be possible to obtain. The most popular combinations include functional (PET, SPECT) with morphological imaging modalities (CT, MRI) that provide the anatomical landmarks of biochemical processes. Table 6 presents a short overview of the main characteristics of single imaging modalities.

Most often, imaging is only possible through the administration of intermediates known as imaging probes (a specific group of biomarkers), which can be simple molecules such as sodium iodide (I-131) for thyroid scintigraphy or macromolecules, such as labeled peptides as DOTATOC labeled with Ga-68 for PET imaging of tumors expressing somatostatin receptors [42]. They should be able to depict the biochemical processes of interest and to be detected externally by suitable imaging devices. It is important that imaging probes should be administered in tracer amounts so as not to induce pharmacological effects or interfere with the biological processes under study. The ideal imaging probe for any specific process should have the following characteristics: specific uptake by the target tissue/organ involved in the process being studied, ability to increase the signal-to-background ratio, ability to accumulate in the target long enough for image acquisition, presents a simple chemistry, widely available, and low cost. At the moment, there is no imaging probe with all these characteristics. However, important efforts are being made by a huge number of research groups and industrial entities regarding a broad range of products, with both diagnostic and therapeutic, or even 'theranostic' (so-called due to the ability to play a role in both fields), characteristics.

\section{Conclusions and Future Trends}

Small-animal imaging has driven biomedical research to a superior level, and are considered high throughput techniques with inherent tremendous advantages over the more conventional ex vivo techniques, with a clear emphasis on real-time monitoring, on being non-destructive and noninvasive, allowing the provision of multiple time points over a wide timescale (longitudinal studies), and multilevel information (from cellular and molecular level to organ and entire organism level), with the additional advantage that each animal can serve as its own control. In fact, the development and dissemination of small-animal imaging equipment and techniques have contributed to a very significant reduction in the number of animals required for scientific research, fully complying with 3R (Replacement, Reduction and Refinement) polices and strategies. The most relevant applications are related to new drug discovery and development; target identification; characterization of drug pharmacological, pharmacokinetic, and pharmacodynamic profiles (including safety and validation studies); and organ function and metabolism in a variety of pathological contexts. The wide recognition of all those advantages has driven the increasing use of small-animal imaging techniques by pharmaceutical companies, contract research organizations, and research institutions. A recent report estimates an annual growth rate of the preclinical imaging market to be about $14.5 \%$ over the next 5 years, reaching the value of \$US1.55 billion dollars in 2017 [132].

Each imaging modality has intrinsic advantages and limitations, providing different kinds of information, most often complementary. While nuclear modalities are suited to providing essentially molecular and functional information, CTs and MRIs provide images with superb spatial resolution. The combination of the so-called functional with morphological imaging modalities has proven very advantageous, providing more accurate and useful information. PET is by far the most sensitive modality, able to detect labeled molecules in picomolar concentrations. In fact, preclinical PET is more often used in the form of multimodality imaging, occupying first and second place in the ranking of the most applied combined techniques with PET-CT and PET-MRI, respectively [50]. The combination of high-resolution modalities, such as microCT or micro-MRI, with highly sensitive techniques providing functional information, such as micro-PET or 
micro-SPECT, will continue to broaden the horizons of research in such key areas as infection, oncology, cardiology, and neurology, contributing not only to expanding the understanding of the underlying mechanisms of disease, but also to the provision of efficient tools for evaluating new therapeutic agents. The added value of multimodality or hybrid imaging approaches has driven its wide acceptance and widespread application over the last years. In the near future, hardware and software levels will further develop, broadening the range of applications and image quality. Although SPECT is less sensitive than PET, its radiolabeling chemistry is usually simpler and easier, including radionuclides with a variety of photonic energies and half-lives, making possible the simultaneous acquisition of multiple probes, labeled with different radionuclides and thus the assessment of distinct molecular events. Compared with PET, the lower cost and wider availability of SPECT agents are important aspects to be considered.

To take full advantage of these techniques, facilities should be carefully planned in order to optimize workflow, while always strictly complying with regulatory issues such as those concerning biosafety, radiation protection, and animal comfort. Staff should be highly specialized and specifically trained to work as well and efficiently with the distinct animal models as with the complexity of the equipment and devices. In this context, successful teams are generally multi-skilled, including researchers with background knowledge as distinct as biology, physiology, veterinary and animal fare, mathematics, physics, electronics, imaging processing, radiochemistry, and pharmaceutical sciences.

\section{References}

1. Milne CP, Kaitin KI. Translational medicine: an engine of change for bringing new technology to community health. Sci Transl Med. 2009 Nov 4;1(5):5cm5.

2. Lewis JS, Achilefu S, Garbow JR, Laforest R, Welch MJ. Small animal imaging: current technology and perspectives for oncological imaging. Eur J Cancer. 2002;38(16):2173-88.
3. Franc BL, Acton PD, Mari C, Hasegawa BH. Small-animal SPECT and SPECT/CT: important tools for preclinical investigation. J Nucl Med. 2008;49(10):1651-63.

4. Allport JR, Weissleder R. In vivo imaging of gene and cell therapies. Exp Hematol. 2001;29(11):1237-46.

5. Pomper MG, Lee JS. Small animal imaging in drug development. Curr Pharm Des. 2005;11(25):3247-72.

6. Deng WP, Wu CC, Lee CC, Yang WK, Wang HE, Liu RS, et al. Serial in vivo imaging of the lung metastases model and gene therapy using HSV1-tk and ganciclovir. J Nucl Med. 2006; 47(5):877-84.

7. Turetschek K, Floyd E, Helbich T, Roberts TP, Shames DM, Wendland MF, et al. MRI assessment of microvascular characteristics in experimental breast tumors using a new blood pool contrast agent (MS-325) with correlations to histopathology. J Magn Reson Imaging. 2001;14(3):237-42.

8. Cheng Z, Mahmood A, Li H, Davison A, Jones AG. [99mTcOAADT]-(CH2)2-NEt2: a potential small-molecule single-photon emission computed tomography probe for imaging metastatic melanoma. Cancer Res. 2005;65(12):4979-86.

9. Gambhir SS, Czernin J, Schwimmer J, Silverman DH, Coleman RE, Phelps ME. A tabulated summary of the FDG PET literature. J Nucl Med. 2001;42(5 Suppl):1S-93S.

10. Bading JR, Shields AF. Imaging of cell proliferation: status and prospects. J Nucl Med. 2008;49(Suppl 2):64S-80S.

11. Blasberg R. PET imaging of gene expression. Eur J Cancer. 2002;38(16):2137-46.

12. Gambhir SS, Herschman HR, Cherry SR, Barrio JR, Satyamurthy $\mathrm{N}$, Toyokuni $\mathrm{T}$, et al. Imaging transgene expression with radionuclide imaging technologies. Neoplasia. 2000;2(1-2): 118-38.

13. Orlova A, Nilsson FY, Wikman M, Widstrom C, Stahl S, Carlsson J, et al. Comparative in vivo evaluation of technetium and iodine labels on an anti-HER2 affibody for single-photon imaging of HER2 expression in tumors. J Nucl Med. 2006; 47(3):512-9.

14. Foss CA, Mease RC, Fan H, Wang Y, Ravert HT, Dannals RF, et al. Radiolabeled small-molecule ligands for prostate-specific membrane antigen: in vivo imaging in experimental models of prostate cancer. Clin Cancer Res. 2005;11(11):4022-8.

15. Haubner RH, Wester HJ, Weber WA, Schwaiger M. Radiotracer-based strategies to image angiogenesis. Q J Nucl Med. 2003;47(3):189-99.

16. Jia B, Shi J, Yang Z, Xu B, Liu Z, Zhao H, et al. 99mTc-labeled cyclic RGDfK dimer: initial evaluation for SPECT imaging of glioma integrin alphavbeta3 expression. Bioconjugate Chem. 2006;17(4):1069-76.

17. Niu G, Chen X. PET imaging of angiogenesis. PET Clin. 2009;4(1):17-38.

18. Holland JP, Lewis JS, Dehdashti F. Assessing tumor hypoxia by positron emission tomography with Cu-ATSM. Q J Nucl Med Mol Imag. 2009;53(2):193-200.

19. Madar I, Huang Y, Ravert H, Dalrymple SL, Davidson NE, Isaacs JT, et al. Detection and quantification of the evolution dynamics of apoptosis using the PET voltage sensor $18 \mathrm{~F}-$ fluorobenzyl triphenyl phosphonium. J Nucl Med. 2009; 50(5):774-80.

20. Murakami Y, Takamatsu H, Taki J, Tatsumi M, Noda A, Ichise $\mathrm{R}$, et al. 18F-labelled annexin V: a PET tracer for apoptosis imaging. Eur J Nucl Med Mol Imag. 2004;31(4):469-74.

21. Jaffer FA, Weissleder R. Seeing within: molecular imaging of the cardiovascular system. Circ Res. 2004;94(4):433-45.

22. Lamb HJ, van der Meer RW, de Roos A, Bax JJ. Cardiovascular molecular MR imaging. Eur J Nucl Med Mol Imag. 2007; 34(Suppl 1):S99-104. 
23. Hua J, Dobrucki LW, Sadeghi MM, Zhang J, Bourke BN, Cavaliere $\mathrm{P}$, et al. Noninvasive imaging of angiogenesis with a $99 \mathrm{mTc}$-labeled peptide targeted at alphavbeta3 integrin after murine hindlimb ischemia. Circulation. 2005;111(24):3255-60.

24. Khaw BA, Tekabe Y, Johnson LL. Imaging experimental atherosclerotic lesions in ApoE knockout mice: enhanced targeting with Z2D3-anti-DTPA bispecific antibody and 99mTc-labeled negatively charged polymers. J Nucl Med. 2006;47(5):868-76.

25. Kolodgie FD, Petrov A, Virmani R, Narula N, Verjans JW, Weber DK, et al. Targeting of apoptotic macrophages and experimental atheroma with radiolabeled annexin V: a technique with potential for noninvasive imaging of vulnerable plaque. Circulation. 2003;108(25):3134-9.

26. Schafers M, Riemann B, Kopka K, Breyholz HJ, Wagner S, Schafers KP, et al. Scintigraphic imaging of matrix metalloproteinase activity in the arterial wall in vivo. Circulation. 2004; 109(21):2554-9.

27. Acton PD, Kung HF. Small animal imaging with high resolution single photon emission tomography. Nucl Med Biol. 2003;30(8):889-95.

28. Lancelot S, Zimmer L. Small-animal positron emission tomography as a tool for neuropharmacology. Trend Pharmacol Sci. 2010;31(9):411-7.

29. Zanzonico P. Noninvasive imaging for supporting basic research. In: Kiessling F, Pichler BJ, editors. Small animal imaging-basics and practical guide. Heidelberg: Springer; 2011. p. 3-16.

30. Kagadis GC, Loudos G, Katsanos K, Langer SG, Nikiforidis GC. In vivo small animal imaging: current status and future prospects. Med Phys. 2010;37(12):6421-42.

31. Weissleder R, Mahmood U. Molecular imaging. Radiology. 2001;219(2):316-33.

32. Massoud TF, Gambhir SS. Molecular imaging in living subjects: seeing fundamental biological processes in a new light. Genes Dev. 2003;17(5):545-80.

33. Grassi R, Lagalla R, Rotondo A. Genomics, proteomics, MEMS and SAIF: which role for diagnostic imaging? La Radiologia medica. 2008;113(6):775-8.

34. Alberti C. From molecular imaging in preclinical/clinical oncology to theranostic applications in targeted tumor therapy. Eur Rev Med Pharmacol Sci. 2012;16(14):1925-33.

35. Meikle SR, Kench P, Kassiou M, Banati RB. Small animal SPECT and its place in the matrix of molecular imaging technologies. Phys Med Biol. 2005;50(22):R45-61.

36. Peterson TE, Shokouhi S. Advances in preclinical SPECT instrumentation. J Nucl Med. 2012;53(6):841-4.

37. Khalil MM, Tremoleda JL, Bayomy TB, Gsell W. Molecular SPECT imaging: an overview. Int J Mol Imaging. 2011;2011: 796025.

38. Peterson TE, Furenlid LR. SPECT detectors: the Anger Camera and beyond. Phys Med Biol. 2011;56(17):R145-82.

39. Kim H, Furenlid LR, Crawford MJ, Wilson DW, Barber HB, Peterson TE, et al. SemiSPECT: a small-animal single-photon emission computed tomography (SPECT) imager based on eight cadmium zinc telluride (CZT) detector arrays. Med Phys. 2006;33(2):465-74.

40. Phelps ME. Positron emission tomography provides molecular imaging of biological processes. Proc Nat Acad Sci USA. 2000;97(16):9226-33.

41. Phelps ME. PET: the merging of biology and imaging into molecular imaging. J Nucl Med. 2000;41(4):661-81.

42. Kowalski J, Henze M, Schuhmacher J, Mäcke HR, Hofmann M, Haberkorn U. Evaluation of Positron Emission Tomography Imaging Using [68 Ga]-DOTA-D Phe1-Tyr3-Octreotide in Comparison to [111In]-DTPAOC SPECT. First Results in
Patients with Neuroendocrine Tumors. Mol Imaging Biol. 2003;5(1):42-8.

43. Holland JP, Sheh Y, Lewis JS. Standardized methods for the production of high specific-activity zirconium-89. Nucl Med Biol. 2009;36(7):729-39.

44. Basu S, Urhan M, Rosenbaum J, Alavi A. PET and PET/CT in the management of thyroid cancer. Method Mol Biol. 2011;727:205-24.

45. Fass L. Imaging and cancer: a review. Mol Oncol. 2008; 2(2):115-52.

46. Levin CS, Zaidi H. Current trends in preclinical PET system design. PET Clin. 2007;2(2):125-60.

47. Yao R, Lecomte R, Crawford ES. Small-animal PET: what is it, and why do we need it? J Nucl Med Technol. 2012 Sep;40(3): 157-65.

48. Hutchins GD, Miller MA, Soon VC, Receveur T. Small animal PET imaging. ILAR J. 2008;49(1):54-65.

49. Lecomte R. Technology challenges in small animal PET imaging. Nucl Instrum Methods Phys Res A. 2004;527(1-2): 157-65.

50. Comley J. In vivo preclinical imaging: an essential tool in translational research. Drug Discovery World. 2011:58-71.

51. Beekman F, van der Have F. The pinhole: gateway to ultra-highresolution three-dimensional radionuclide imaging. Eur J Nucl Med Mol Imaging. 2007;34(2):151-61.

52. Shao Y, Cherry SR, Farahani K, Slates R, Silverman RW, Meadors K, et al. Development of a PET detector system compatible with MRI/NMR systems. IEEE Trans Nucl Sci. 1997;44(3):1167-71.

53. Tsui BM, Kraitchman DL. Recent advances in small-animal cardiovascular imaging. J Nucl Med. 2009;50(5):667-70.

54. de Kemp RA, Epstein FH, Catana C, Tsui BM, Ritman EL. Small-animal molecular imaging methods. J Nucl Med. 2010; 1(51 Suppl 1): 18S-32S.

55. Fleming JS, Alaamer AS. Influence of collimator characteristics on quantification in SPECT. J Nucl Med. 1996;37(11):1832-6.

56. Chatziioannou AF, Cherry SR, Shao Y, Silverman RW, Meadors K, Farquhar TH, et al. Performance evaluation of microPET: a high-resolution lutetium oxyorthosilicate PET scanner for animal imaging. J Nucl Med. 1999;40(7):1164-75.

57. Levin CS, Hoffman EJ. Calculation of positron range and its effect on the fundamental limit of positron emission tomography system spatial resolution. Phys Med Biol. 1999;44(3):781.

58. Tai YC, Ruangma A, Rowland D, Siegel S, Newport DF, Chow PL, et al. Performance evaluation of the microPET focus: a third-generation microPET scanner dedicated to animal imaging. J Nucl Med. 2005;46(3):455-63.

59. Beekman FJ, van der Have F, Vastenhouw B, van der Linden AJ, van Rijk PP, Burbach JP, et al. U-SPECT-I: a novel system for submillimeter-resolution tomography with radiolabeled molecules in mice. J Nucl Med. 2005;46(7):1194-200.

60. Vastenhouw B, Beekman F. Submillimeter total-body murine imaging with U-SPECT-I. J Nucl Med. 2007;48(3):487-93.

61. Chatziioannou AF. Instrumentation for molecular imaging in preclinical research: Micro-PET and Micro-SPECT. Proc Am Thorac Soc. 2005;2(6):533-6, 10-11.

62. Henriksen G, Drzezga A. Imaging in neurology research II: PET imaging in CNS disorders. In: Kiessling F, Pichler BJ, editors. Small animal imaging — basics and practical guide. Heidelberg: Springer; 2011. p. 499-513.

63. Hume SP, Gunn RN, Jones T. Pharmacological constraints associated with positron emission tomographic scanning of small laboratory animals. Eur J Nucl Med. 1998;25(2):173-6.

64. Judenhofer MS, Wiehr S, Kukuk D, Fischer K, Pichler BJ. Guidelines for nuclear image analysis. In: Kiessling F, Pichler 
BJ, editors. Small animal imaging-basics and practical guide. Heidelberg: Springer; 2011. p. 379-86.

65. Cavanaugh D, Johnson E, Price RE, Kurie J, Travis EL, Cody DD. In vivo respiratory-gated micro-CT imaging in small-animal oncology models. Mol Imaging. 2004;3(1):55-62.

66. Ritman EL. Current status of developments and applications of micro-CT. Annu Rev Biomed Eng. 2011;15(13):531-52.

67. Badea CT, Drangova M, Holdsworth DW, Johnson GA. In vivo small-animal imaging using micro-CT and digital subtraction angiography. Phys Med Biol. 2008;53(19):R319-50.

68. Lee N, Choi SH, Hyeon T. Nano-sized CT contrast agents. Adv Mater. 2013;25(19):2641-60.

69. Pietsch H. CT contrast agents. In: Kiessling F, Pichler BJ, editors. Small animal imaging-basics and practical guide. Heidelberg: Springer; 2011. p. 141-9.

70. Kalender WA, Deak P, Engelke K, Karolczak M. X-ray and X-ray CT. In: Kiessling F, Pichler BJ, editors. Small animal imaging - basics and practical guide. Heidelberg: Springer; 2011. p. 125-39.

71. Dufort S, Sancey L, Wenk C, Josserand V, Coll JL. Optical small animal imaging in the drug discovery process. Biochim Biophys Acta. 2010;1798(12):2266-73.

72. Tremoleda JL, Khalil M, Gompels LL, Wylezinska-Arridge M, Vincent $\mathrm{T}$, Gsell W. Imaging technologies for preclinical models of bone and joint disorders. EJNMMI Res. 2011;1(1):11.

73. Koba W, Kim K, Lipton ML, Jelicks L, Das B, Herbst L, et al. Imaging devices for use in small animals. Semin Nucl Med. 2011;41(3):151-65.

74. Jakob P. Small animal magnetic resonance imaging: basic principles, instrumentation and practical issues. In: Kiessling F, Pichler BJ, editors. Small animal imaging-basics and practical guide. Heidelberg: Springer; 2011. p. 151-64.

75. Brockmann MA. Use of clinical MR scanners for small rodent imaging. Methods. 2007;43(1):1.

76. Leroy-Willig A, Geldwerth-Feniger G. Nuclear magnetic resonance imaging and spectroscopy. In: Ntziachristos V, LeroyWillig A, Tavitian B, editors. Textbook of in vivo imaging in vertebrates. UK: Wiley; 2007. p. 1-56.

77. Weber WA, Kiessling F. Imaging in oncology research. In: Kiessling F, Pichler BJ, editors. Small animal imaging-basics and practical guide. Heidelberg: Springer; 2011. p. 543-64.

78. Schaefer PW, Grant PE, Gonzalez RG. Diffusion-weighted MR imaging of the brain. Radiology. 2000;217(2):331-45.

79. Heeger DJ, Ress D. What does fMRI tell us about neuronal activity? Nat Rev Neurosci. 2002;3(2):142-51.

80. Goetti R, O'Gorman R, Khan N, Kellenberger CJ, Scheer I. Arterial spin labelling MRI for assessment of cerebral perfusion in children with moyamoya disease: comparison with dynamic susceptibility contrast MRI. Neuroradiology. 2013;55(5):639-47.

81. Cutajar M, Thomas DL, Banks T, Clark CA, Golay X, Gordon I. Repeatability of renal arterial spin labelling MRI in healthy subjects. MAGMA. 2012;25(2):145-53.

82. Thomas D, Wells J. MR angiography and arterial spin labelling. Method Mol Biol. 2011;711:327-45.

83. Kazan SM, Chappell MA, Payne SJ. Modelling the effects of cardiac pulsations in arterial spin labelling. Phys Med Biol. 2010;55(3):799-816.

84. Richards TL. Multinuclear Magnetic Resonance Spectroscopic Imaging. Encyclopedia of Analytical Chemistry. New York: Wiley; 2006.

85. Rudin M. Imaging techniques. Molecular imaging: basic principles and applications in biomedical research. London: Imperial College Press; 2005. p. 45-140.

86. Rosen Y, Lenkinski RE. Recent advances in magnetic resonance neurospectroscopy. Neurotherapeutics. 2007;4(3):330-45.
87. Gujar SK, Maheshwari S, Bjorkman-Burtscher I, Sundgren PC. Magnetic resonance spectroscopy. J Neuroophthalmol. 2005; 25(3):217-26.

88. van der Graaf M. In vivo magnetic resonance spectroscopy: basic methodology and clinical applications. Eur Biophys J. 2010;39(4):527-40.

89. Zhu H, Barker PB. MR spectroscopy and spectroscopic imaging of the brain. Methods Mol Biol. 2011;711:203-26.

90. Forster D, Davies K, Williams S. Magnetic resonance spectroscopy in vivo of neurochemicals in a transgenic model of Alzheimer's disease: a longitudinal study of metabolites, relaxation time, and behavioral analysis in TASTPM and wildtype mice. Magn Reson Med. 2013;69(4):944-55.

91. He Q, Xu RZ, Shkarin P, Pizzorno G, Lee-French CH, Rothman DL, et al. Magnetic resonance spectroscopic imaging of tumor metabolic markers for cancer diagnosis, metabolic phenotyping, and characterization of tumor microenvironment. Dis Markers. 2003;19(2-3):69-94.

92. Bremer C, Ntziachristos V, Weissleder R. Optical-based molecular imaging: contrast agents and potential medical applications. Eur Radiol. 2003;13(2):231-43.

93. Chin PT, Welling MM, Meskers SC, Valdes Olmos RA, Tanke $\mathrm{H}$, van Leeuwen FW. Optical imaging as an expansion of nuclear medicine: Cerenkov-based luminescence vs fluorescence-based luminescence. Eur J Nucl Med Mol Imaging. 2013; 40(8):1283-91.

94. Wilson T, Hastings J. Bioluminescence. Annu Rev Cell Dev Biol. 1988;14:197-230.

95. Greer LF III, Szalay AA. Imaging of light emission from the expression of luciferases in living cells and organisms: a review. Luminescence. 2002;17(1):43-74.

96. Weissleder R. Scaling down imaging: molecular mapping of cancer in mice. Nat Rev Cancer. 2002;2(1):11-8.

97. Culver J, Akers W, Achilefu S. Multimodality molecular imaging with combined optical and SPECT/PET modalities. J Nucl Med. 2008;49(2):169-72.

98. Ntziachristos V, Bremer C, Weissleder R. Fluorescence imaging with near-infrared light: new technological advances that enable in vivo molecular imaging. Eur Radiol. 2003;13(1):195-208.

99. Kruger RA. Photoacoustic ultrasound. Med Phys. 1994;21(1): 127-31.

100. Wang LV, Hu S. Photoacoustic tomography: in vivo imaging from organelles to organs. Science. 2012;335(6075): $1458-62$.

101. Robertson R, Germanos MS, Li C, Mitchell GS, Cherry SR, Silva MD. Optical imaging of Cerenkov light generation from positron-emitting radiotracers. Phys Med Biol. 2009;54(16): N355-65.

102. Liu H, Ren G, Miao Z, Zhang X, Tang X, Han P, et al. Molecular optical imaging with radioactive probes. PloS One. 2010;5(3):e9470.

103. Spinelli AE, Ferdeghini M, Cavedon C, Zivelonghi E, Calandrino R, Fenzi A, et al. First human Cerenkography. J Biomed Opt. 2013;18(2):20502.

104. Vooijs M, Jonkers J, Lyons S, Berns A. Noninvasive imaging of spontaneous retinoblastoma pathway-dependent tumors in mice. Cancer Res. 2002;62(6):1862-7.

105. Liang HD, Blomley MJ. The role of ultrasound in molecular imaging. British J Radiol. 2003;76 Spec No 2:S140-50.

106. Coatney RW. Ultrasound imaging: principles and applications in rodent research. ILAR J. 2001;42(3):233-47.

107. Tremoleda JL, Kerton A, Gsell W. Anaesthesia and physiological monitoring during in vivo imaging of laboratory rodents: considerations on experimental outcomes and animal welfare. EJNMMI Res. 2012;2(1):44. 
108. Skresanova IV, Barannik EA. Correlation functions and power spectra of Doppler response signals in ultrasonic medical applications. Ultrasonics. 2012;52(5):676-84.

109. Deshpande N, Needles A, Willmann JK. Molecular ultrasound imaging: current status and future directions. Clin Radiol. 2010;65(7):567-81.

110. Greco A, Mancini M, Gargiulo S, Gramanzini M, Claudio PP, Brunetti A, et al. Ultrasound biomicroscopy in small animal research: applications in molecular and preclinical imaging. J Biomed Biotechnol. 2012;2012:519238.

111. Golden HB, Sunder S, Liu Y, Peng X, Dostal DE. In utero assessment of cardiovascular function in the embryonic mouse heart using high-resolution ultrasound biomicroscopy. Method Mol Biol. 2012;843:245-63.

112. Cheung AM, Brown AS, Cucevic V, Roy M, Needles A, Yang $\mathrm{V}$, et al. Detecting vascular changes in tumour xenografts using micro-ultrasound and micro-ct following treatment with VEGFR-2 blocking antibodies. Ultrasound Med Biol. 2007;33(8): 1259-68.

113. Kaufmann BA, Lankford M, Behm CZ, French BA, Klibanov $\mathrm{AL}, \mathrm{Xu} \mathrm{Y}$, et al. High-resolution myocardial perfusion imaging in mice with high-frequency echocardiographic detection of a depot contrast agent. J Am Soc Echocardiogr. 2007;20(2): $136-43$.

114. Alves KZ, Soletti RC, de Britto MA, de Matos DG, Soldan M, Borges HL, et al. In Vivo endoluminal ultrasound biomicroscopic imaging in a mouse model of colorectal cancer. Acad Radiol. 2013;20(1):90-8.

115. Alexandrakis G, Rannou FR, Chatziioannou AF. Effect of optical property estimation accuracy on tomographic bioluminescence imaging: simulation of a combined optical-PET (OPET) system. Phys Med Biol. 2006;51(8):2045-53.

116. Peter J, Ruehle H, Stamm V, Schulz RB, Smith MF, Welch B, et al. Development and initial results of a dual-modality SPECT/ optical small animal imager. Nuclear Science Symposium Conference Record; 2005 IEEE; 2005 23-29 Oct: p. 4.

117. Hyde D, de Kleine R, MacLaurin SA, Miller E, Brooks DH, Krucker T, et al. Hybrid FMT-CT imaging of amyloid-beta plaques in a murine Alzheimer's disease model. Neuroimage. 2009;44(4):1304-11.

118. Wen Z, Fahrig R, Williams ST, Pelc NJ. Shimming with permanent magnets for the $\mathrm{x}$-ray detector in a hybrid $\mathrm{x}$-ray/ MR system. Med Phys. 2008;35(9):3895-902.

119. Gulsen G, Birgul O, Unlu MB, Shafiiha R, Nalcioglu O. Combined diffuse optical tomography (DOT) and MRI system for cancer imaging in small animals. Technol Cancer Res Treat. 2006;5(4):351-63.

120. Kundu BK, Stolin AV, Pole J, Baumgart L, Fontaine M, Wojcik $\mathrm{R}$, et al. Tri-modality small animal imaging system. IEEE Trans Nucl Sci. 2006;53(1):66-70.

121. Veit-Haibach P, Kuhn FP, Wiesinger F, Delso G, von Schulthess G. PET-MR imaging using a tri-modality PET/CT-MR system with a dedicated shuttle in clinical routine. MAGMA. 2013; 26(1):25-35

122. Tsukamoto E, Ochi S. PET/CT today: system and its impact on cancer diagnosis. Ann Nucl Med. 2006;20(4):255-67.

123. Bergeron M, Cadorette J, Beaudoin JF, Lepage MD, Robert G, Selivanov V, et al. Performance evaluation of the LabPET APD- based digital PET scanner. IEEE Trans Nucl Sci. 2009;56(1): $10-6$.

124. Levin Klausen T, Hogild Keller S, Vinter Olesen O, Aznar M, Andersen FL. Innovations in PET/CT. Q J Nucl Med Mol Imaging. 2012;56(3):268-79.

125. Beyer T, Freudenberg LS, Townsend DW, Czernin J. The future of hybrid imaging-part 1: hybrid imaging technologies and SPECT/CT. Insight Imaging. 2011;2(2):161-9.

126. Hammer BE, Christensen NL, Heil BG. Use of a magnetic field to increase the spatial resolution of positron emission tomography. Med Phys. 1994;21(12):1917-20.

127. Beyer T, Freudenberg LS, Czernin J, Townsend DW. The future of hybrid imaging-part 3: PET/MR, small-animal imaging and beyond. Insight Imaging. 2011;2(3):235-46.

128. Wirrwar A, Vosberg H, Herzog H, Halling H, Weber S, MullerGartner HW. 4.5 Tesla magnetic field reduces range of highenergy positrons-potential implications for positron emission tomography. IEEE Trans Nucl Sci. 1997;44(2):184-9.

129. Cherry SR. Multimodality imaging: beyond PET/CT and SPECT/CT. Semin Nucl Med. 2009;39(5):348-53.

130. Wagenknecht G, Kaiser HJ, Mottaghy FM, Herzog H. MRI for attenuation correction in PET: methods and challenges. MAGMA. 2013;26(1):99-113.

131. Tartis MS, Kruse DE, Zheng H, Zhang H, Kheirolomoom A, Marik J, et al. Dynamic microPET imaging of ultrasound contrast agents and lipid delivery. J Controlled Release. 2008; 131(3):160-6.

132. Markets, Markets. Small Animal Imaging (In Vivo) Market: Competitive Analysis \& Global Forecasts to 2017. 2012 [cited 16 Feb 2013]. http://www.companiesandmarkets.com/Market/ Healthcare-and-Medical/Market-Research/Small-Animal-Imaging-In-Vivo-Market-Competitive-Analysis-Global-Forecasts-to2017/RPT1134167.

133. Balcerzyk M, Kontaxakis G, Delgado M, Garcia-Garcia L, Correcher C, Gonzalez AJ, et al. Initial performance evaluation of a high resolution Albira small animal positron emission tomography scanner with monolithic crystals and depth-ofinteraction encoding from a user's perspective. Measurement Sci Technol. 2009;20(10).

134. Szanda I, Mackewn J, Patay G, Major P, Sunassee K, Mullen GE, et al. National electrical manufacturers association NU-4 performance evaluation of the PET component of the NanoPET/ CT preclinical PET/CT Scanner. J Nucl Med. 2011;52(11): 1741-7.

135. Goorden MC, van der Have F, Kreuger R, Ramakers RM, Vastenhouw B, Burbach JP, et al. VECTor: a preclinical imaging system for simultaneous submillimeter SPECT and PET. J Nucl Med. 2013;54(2):306-12.

136. Goertzen AL, Bao QN, Bergeron M, Blankemeyer E, Blinder S, Canadas M, et al. NEMA NU 4-2008 comparison of preclinical PET imaging systems. J Nucl Med. 2012;53(8):1300-9.

137. Bao Q, Newport D, Chen M, Stout DB, Chatziioannou AF. Performance evaluation of the inveon dedicated PET preclinical tomograph based on the NEMA NU-4 standards. J Nucl Med. 2009;50(3):401-8.

138. Herrmann K, Dahlbom M, Nathanson D, Wei L, Radu C, Chatziioannou A, et al. Evaluation of the Genisys4, a bench-top preclinical PET scanner. J Nucl Med. 2013;54(7):1162-7. 